\title{
Which Cue to "Want?" Central Amygdala Opioid Activation Enhances and Focuses Incentive Salience on a Prepotent Reward Cue
}

\author{
Stephen V. Mahler and Kent C. Berridge \\ Department of Psychology, University of Michigan, Ann Arbor, Michigan 48109
}

The central nucleus of the amygdala (CeA) helps translate learning into motivation, and here, we show that opioid stimulation of CeA magnifies and focuses learned incentive salience onto a specific reward cue (pavlovian conditioned stimulus, or CS). This motivation enhancement makes that cue more attractive, noticeable, and liable to elicit appetitive and consummatory behaviors. To reveal the focusing of incentive salience, we exploited individual differences in an autoshaping paradigm in which a rat prefers to approach, nibble, and sniff one of two reward-associated stimuli (its prepotent stimulus). The individually prepotent cue is either a predictive CS + that signals reward ( $8 \mathrm{~s}$ metal lever insertion) or instead the metal cup that delivers sucrose pellets (the reward source). Results indicated that CeA opioid activation by microinjection of the $\mu$ agonist DAMGO $(0.1 \mu \mathrm{g})$ selectively and reversibly enhanced the attractiveness of whichever reward CS was that rat's prepotent cue. CeA DAMGO microinjections made rats more vigorously approach their particular prepotent CS and to energetically sniff and nibble it in a nearly frenzied consummatory manner. Only the prepotent cue was enhanced as an incentive target, and alternative cues were not enhanced. Conversely, inactivation of CeA by muscimol microinjection $(0.25 \mu \mathrm{g})$ suppressed approach, nibbles, and sniffs of the prepotent CS. Confirming modulation of incentive salience, unconditioned food intake was similarly increased by DAMGO microinjection and decreased by muscimol in CeA. We conclude that opioid neurotransmission in CeA helps determine which environmental stimuli become most "wanted," and how "wanted" they become. This may powerfully guide reward-seeking behavior.

\section{Introduction}

Discrete cues or pavlovian-conditioned stimuli (CSs) that predict rewards (unconditioned stimuli, or UCSs) can potently attract attention, stimulate motivation, and become worked for and "wanted" almost like UCS rewards themselves (Timberlake and Grant; Holland; Bindra, 1978; Toates, 1986; Robbins and Everitt; Gallagher and Schoenbaum, 1999; Berridge, 2001; Di Ciano and Everitt, 2005). This can sometimes lead to bizarre motivated behaviors directed at CSs, rather than at reward UCSs themselves. For example, crack cocaine addicts are known to "chase ghosts," or compulsively pick up pebbles on the ground that resemble crack rocks (Rosse et al., 1993; Berridge, 2007), rats eagerly sniff a lever CS that predicts cocaine or brain stimulation reward (Peterson et al., 1972; Uslaner et al., 2006), quail copulate with a birdsized terrycloth CS that predicts a sex partner (Burns and Domjan, 1996), and rats and pigeons bite, lick, or peck CS levers or lighted keys for food or water almost as if the CSs were the asso-

Received Aug. 14, 2008; revised March 24, 2009; accepted April 3, 2009.

Funding was provided by National Institutes of Health Grants DA015188 and MH63649 (K.C.B.) and National Research Service Award DA021481 (S.V.M.). We acknowledge Michelle DiMondo and Phillip Hoberg for assistance with histology. We thank Terry Robinson, Wayne Aldridge, and Sarah Newman for helpful discussion and comments on an earlier version of this manuscript.

Correspondence should be addressed to Dr. Stephen V. Mahler at his present address: Medical University of South Carolina, 409 BSB173 Ashley Avenue, Charleston, SC 29425. E-mail: mahler@musc.edu.

DOI:10.1523/JNEUROSCI.3875-08.2009

Copyright $\odot 2009$ Society for Neuroscience $\quad 0270-6474 / 09 / 296500-14 \$ 15.00 / 0$ ciated ingestive rewards themselves (Jenkins and Moore, 1973; Boakes, 1977; Tomie et al., 2008).

One explanation for such peculiar consummatory behaviors focused on CSs is provided by the incentive salience hypothesis of motivation, which posits that mesocorticolimbic activation bestows incentive salience on selected reward cues, guided by previous pavlovian associations (Robinson and Berridge, 1993; Berridge, 2001, 2007). The cues take on incentive motivational features of their rewards (Bindra, 1978; Toates, 1986; Berridge, 2001). As a consequence, those cues become attractive, "wanted" incentives themselves, as well as triggers of "wanting" for associated UCS rewards. However, some cues and rewards become more "wanted" than others, and "wanting" can sometimes be focused compulsively on one reward cue at a time, as in addiction.

How is incentive salience generated and focused onto a particular reward cue? Activation of mesocorticolimbic circuits at the moment of cue reencounter must be guided by previous associations with rewards and attributed to a selected perceived cue or representation. The amygdala is a potential brain structure in which pavlovian learning could be translated into motivational salience and focused on a particular percept (Morris and Dolan, 2001; Di Ciano and Everitt, 2004; Holland and Gallagher, 2004; Corbit and Balleine; Phelps and LeDoux, 2005; Balleine and Killcross, 2006; Beaver et al., 2006; Phillips et al., 2008). Opioid neurotransmission in the CeA nucleus may be especially important for generating such incentive motivation (Gosnell, 1988; Kim et al., 2004; Scott et al., 2007). 


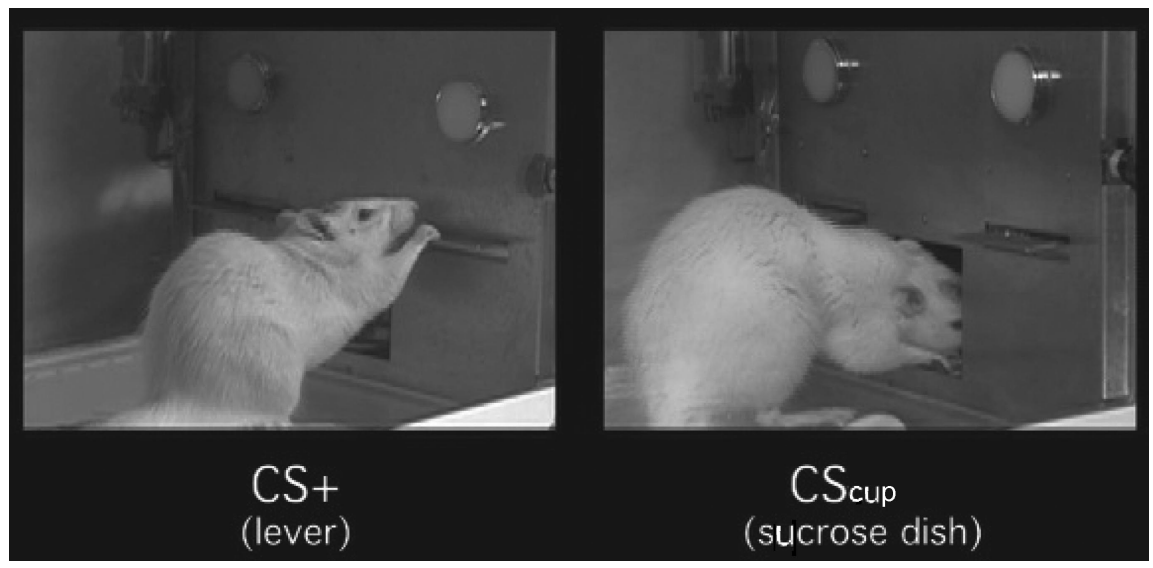

Figure 1. Examples of appetitive- consummatory behaviors directed at CS + . Each rat directs cue-triggered consummatory behaviors toward its own prepotent $C S$ : either the predictive $C S+$ lever or the sucrose-delivering $C S_{\text {cup. }}$. The target of incentive salience differs across individuals in this autoshaping paradigm, although in all cases the extension of the CS + lever triggers a phasic peak of CS "wanting" and appetitive-consummatory behaviors. Please visit http://www.Isa.umich.edu/psych/ research\&labs/berridge/multimediavideo/which_cue_to_want.mov, or see Video 1, available at www.jneurosci.org as supplemental material to see examples of these behaviors.

Here, we predicted that activating opioid receptors in CeA would enhance both the focus and the intensity of incentive salience, making a preselected CS more "wanted" than others. We used microinjections of a $\mu$ agonist to activate CeA opioid circuits and muscimol microinjections to more generally inactivate the CeA. We used a pavlovian autoshaping or sign-tracking paradigm to test whether CeA controls the direction and intensity of appetitive-consummatory behaviors and confirmed up/ down modulation of incentive salience with food-intake tests. Our results indicate that amygdala circuits can powerfully enhance and focus incentive salience on a CS to determine which cue to "want."

\section{Materials and Methods Overview}

When a metal CS + lever is repeatedly inserted through a wall to predict a sucrose pellet delivered into a nearby metal cup (which we will call the $\mathrm{CS}_{\text {cup }}$ ), a rat may come to approach, nibble, sniff, and bite either the predictive CS+ lever or the sucrose-delivering $\mathrm{CS}_{\text {cup }}$, but usually not both equally (Hearst and Jenkins, 1974; Boakes, 1977; Flagel et al., 2007, 2009). That is, each rat develops its own individual prepotent CS to preferentially approach and "consume," a choice that is correlated with particular mesolimbic markers that differ across individuals (Flagel et al., $2007,2008,2009)$. Once the preference emerges for an individual rat, it tends not to change. We will call whichever CS target a rat reliably chose its "prepotent CS" (Valenstein et al., 1970). This choice of a particular prepotent CS is so reliable that a rat can be categorized as either "CS+ lever preferring" or " $\mathrm{CS}$ cup preferring" within several days of training (Flagel et al., 2007, 2008). We predicted that CeA opioid activation by DAMGO microinjection would intensify appetitive-consummatory behaviors directed toward a rat's prepotent CS only, namely whichever cue it came to preferentially approach and interact with during early training. Conversely, we predicted that GABAergic inactivation of the CeA via muscimol microinjection would suppress appetitive-consummatory behaviors directed at the prepotent CS. These predictions were confirmed using detailed video analyses of consummatory behaviors directed at CSs in an autoshaping paradigm. We further measured effects on food intake in the same rats to confirm that motivation for the UCS was increased by CeA DAMGO and decreased by CeA inactivation similarly as CS-directed motivation. A Fos plume mapping technique was also used to estimate where microinjected drugs acted anatomically to exert all these behavioral effects.

\section{Subjects}

Sprague Dawley rats were used $(n=105 \mathrm{fe}-$ males, 250-350 g; individual experiments used $n$ s of 24,64 , and 17 as described below, and every estrous phase was included). Rats were housed in a reverse $12 \mathrm{~h}$ light/ $12 \mathrm{~h}$ dark cycle, $\sim 21^{\circ} \mathrm{C}$ room (housed in pairs until surgery and alone thereafter). Chow and water were available ad libitum at all times, except during autoshaping procedures when food was slightly restricted to $20-25 \mathrm{~g} / \mathrm{d} / \mathrm{rat}$ (delivered daily after training or testing). To maximize the impact of microinjections in both behavior tests and in Fos plume analyses, separate groups were used to measure (1) the attribution or expression of food consummatory-like behaviors targeted at CSs (tested during or after learning in different groups), (2) motivation to consume UCS reflected in food intake, and (3) local spread of neuronal modulation mapped by local Fos plumes around sites of DAMGO or muscimol microinjections.

\section{Autoshaping paradigm}

To assess whether CeA activation/inactivation altered appetitive and consummatory behaviors directed toward a reward CS, we measured cue-triggered consumption-like behaviors directed toward the metal lever (CS + lever) that signaled sucrose pellet delivery and the metal cup $\left(\mathrm{CS}_{\text {cup }}\right)$ that delivered it. Consummatory behaviors were sniffing, nibbling, and biting movements directed to the $\mathrm{CS}+$ lever or the $\mathrm{CS}_{\text {cup }}$. Each CS was a discrete and localized physical object that could be grasped and "eaten" (i.e., approximately the size of a chow pellet, located in one place, and easily noticed) (Tomie, 1996). The sequence of nibble-and-sniff ingestive movements targeted at a CS resembles initial ingestive movements directed toward a UCS food pellet at onset of eating (described below; see examples in Figs. 1 and 3).

The CS + was a metal $4.5 \times 2 \mathrm{~cm}$ lever containing a light-emitting diode that was physically inserted through the chamber wall for $8 \mathrm{~s} \mathrm{im}$ mediately before each UCS pellet delivery and accompanied by a $2.9 \mathrm{kHz}$ continuous tone during lever insertion (Flagel et al., 2007, 2009). The CS + presentation was a phasic event that predicted the UCS with $100 \%$ correlation, and the physical lever was discrete, graspable, and bitable (to promote consummatory conditioned responses). The $\mathrm{CS}_{\text {cup }}$ was a recessed dish $(3 \mathrm{~cm}$ diameter $)$ with raised metal edges $(0.7 \mathrm{~cm}$ high) that rats could also approach, sniff, insert their head into, nibble, and bite. Contrasting the two sucrose-associated stimuli, the CS+ lever was the most predictive CS, having the highest correlation with presentations of the UCS reward (but spatially segregated from it by $5 \mathrm{~cm}$ ). The $\mathrm{CS}_{\text {cup }}$ was more immediately proximal in space and more contiguous in time to the UCS pellet (because a rat had to retrieve each UCS directly from the cup at the moment of reward), but less correlated in a predictive sense because of its presence during the entire session, including during absence of CS + or UCS. Extension of the CS+ lever was the informationcontaining, predictive stimulus that triggered cue approach and consummatory behaviors directed at whichever cue was prepotent in a given rat. Finally, a third stimulus (or control CS-) that had no specific excitatory or inhibitory associations was always present in the form of a second lever that remained in the chamber throughout the entire session.

\section{Behavioral videoscoring}

All video analyses were scored in slow motion (1/10 to $1 / 2$ actual speed) by observers blind to experimental conditions. For autoshaping experiments, a video camera positioned under the transparent floor of the autoshaping chamber provided a clear view of the rat's head and body. In addition, presses on the CS + lever and control CS - lever were automatically recorded, and nose pokes into the sucrose cup were recorded by photobeam breaks. Behavior for the $8 \mathrm{~s}$ of the 5th, 10th, 15th, 20th, and 25th CS + presentations of each scored session (and the $8 \mathrm{~s}$ before each cue in the autoshaping expression experiments) was also coded by blind 
observers. Consumption-like nibbles and sniffs of the CS+ lever and $\mathrm{CS}_{\text {cup }}$ were the primary measures of attempted cue consumption. "CS nibbles and sniffs" were small-amplitude, short-duration $(<0.5 \mathrm{~s})$ exploratory movements of the mouth or nose on the lever or sucrose cup, in physical contact with it, typically occurring in bouts of $1-2 \mathrm{~s}$ each (1-3 $\mathrm{Hz}$ ), and resembling the exploratory nibbles and sniffs of a chow pellet UCS before it is eaten. Also similar to food-intake behavior, most rats transitioned from preliminary nibbling and sniffing of the CS to "slow bites" of the CS, in which they grabbed the lever with one or both paws, and clearly opened and closed their mandibles on it $>0.5-1 \mathrm{~s}$ while making tooth contact with it.

In food-intake tests, scored behaviors included time spent eating, number of eating bouts, latency to eat, food sniffing and nibbling bouts, time drinking, number of drinking bouts, occurrences and seconds of defensive paw treading, bouts of digging in bedding, cage crosses, and rears.

\section{Surgery}

To permit microinjections for behavioral testing or Fos, all rats were anesthetized with ketamine $(80 \mathrm{mg} / \mathrm{kg})$, xylazine $(7 \mathrm{mg} / \mathrm{kg})$, and atropine $(0.04 \mathrm{mg} / \mathrm{kg})$ and surgically implanted with chronic, bilateral microinjection guide cannulae (23 ga; $14 \mathrm{~mm}$ long; positioned $2 \mathrm{~mm}$ above CeA sites). Cannulae were anchored to the skull with bone screws and acrylic cement, and steel stylets were inserted to prevent occlusion. Stereotaxic placement coordinates for cranial cannulae were calculated based on Paxinos and Watson (2007). Cannulae were aimed at multiple sites in the CeA ranging from ( $\mathrm{mm}$ relative to bregma), -1.8 to $-3.0 \mathrm{AP} ; \pm 3.0$ to $\pm 4.2 \mathrm{ML}$; and -5.8 to $-6.5 \mathrm{DV}$, or at control sites in nearby amygdaloid structures, including the basolateral amygdala (BLA), basomedial amygdala (BMA), and interstitial nucleus of the posterior limb of the anterior commissure. All rats were allowed 1 week to recover from surgery before any habituation or testing procedures were started.

\section{Behavioral experiment descriptions}

$D A M G O$ enhancement of previously learned CS incentive salience. The first experiment tested whether DAMGO microinjection that was administered on a test day after CS-UCS learning sessions were completed would enhance approach and consummatory-type interactions with a previously learned reward CS. This group of rats was trained drug-free on an autoshaping task for $5 \mathrm{~d}$ after surgery and recovery. Beginning on day 6 , the rats were given bilateral microinjections of DAMGO and vehicle in randomized order $\left(n=24, \mathrm{CS}+\right.$ lever prepotent $n=15 ; \mathrm{CS}_{\text {cup }}$ prepotent $n=9)$ on each of the next two sessions $(0.1 \mu \mathrm{g} / 0.5 \mu \mathrm{l}, 48 \mathrm{~h}$ between test days), immediately before testing in the same autoshaping paradigm. For all microinjections, rats were gently held while stylets were removed, and a microinjector was inserted extending $2 \mathrm{~mm}$ beyond the end of each guide cannulae. Vehicle or drugs were bilaterally infused in a $0.5 \mu \mathrm{l}$ volume over $90 \mathrm{~s}$ using an automated syringe pump. The microinjector was held in place for 1 min after each injection to allow for drug diffusion from the injector tip.

DAMGO enhancements during learning. Another experiment tested whether amygdala opioid stimulation would similarly enhance incentive salience if administered on initial training days immediately before learning. Separate rats were given daily microinjections of either vehicle $(n=$ 22 , CS + lever prepotent $n=14 ; \mathrm{CS}_{\text {cup }}$ prepotent $\left.n=8\right)$ or DAMGO $(0.1$ $\mu \mathrm{g} / 0.5 \mu \mathrm{l}, n=30$, CS + lever prepotent, $n=22 ; \mathrm{CS}_{\text {cup }}$ prepotent, $n=8$ ) into CeA before each of the first six daily autoshaping training sessions.

GABA suppression of prepotent CS incentive salience. To test the opposite prediction that amygdala inactivation would suppress approach and consummatory transactions with a prepotent cue, an additional set of experiments was run using microinjections of the $\mathrm{GABA}_{\mathrm{A}}$ agonist muscimol $[0.25 \mu \mathrm{g} / 0.5 \mu \mathrm{l}$; dose chosen to temporarily inactivate amygdala based on previous reports of behavioral suppression or learning interference (Wilensky et al., 2000; Maren et al., 2001)]. The same rats from the first experiment $(n=24)$ were given seven additional drug-free training days after DAMGO tests (days 8-14) and then tested after microinjections of either muscimol $(0.25 \mu \mathrm{g} / 0.5 \mu \mathrm{l})$ or vehicle in randomized order on days 15 and 16 (held $48 \mathrm{~h}$ apart). Finally, to test whether muscimol inactivation of CeA before training would also prevent a CS from being attributed with incentive salience during learning, a separate group of rats received microinjections of muscimol $(0.25 \mu \mathrm{g} / 0.5 \mu \mathrm{l} ; n=4)$ or vehicle $(n=4)$ before each of the first $4 \mathrm{~d}$ of autoshaping training.

Opioid stimulation and GABA suppression of UCS intake: effects of amygdala opioid stimulation and inactivation on food UCS consumption. To confirm that modulation of CS-directed food consummatory-like behaviors was caused by changes in incentive salience, we tested whether motivation to consume a UCS reward was also altered in the same directions by drug microinjections. Food-intake tests were performed in transparent plastic tub cages with preweighed food (Purina chow pellets), ad libitum water, and corncob bedding. Each rat was tested for $1 \mathrm{~h}$ intake in the same cage each day, after microinjections into amygdala of vehicle and either DAMGO $(0.1 \mu \mathrm{g}, n=22)$ or muscimol $(0.25 \mu \mathrm{g}, n=$ 9 ) in counterbalanced order on separate days $48 \mathrm{~h}$ apart. Food and water intake was measured in grams, and behavior was videotaped for subsequent analysis of coded behaviors.

\section{Autoshaping testing apparatus}

Autoshaping chambers were $30.5 \times 24.1 \times 21.0 \mathrm{~cm}$, with steel front and back plates, and clear plastic sides, ceiling, and floor. A red house light was mounted on the top of the back wall, which was lit throughout all sessions. Two retractable levers were present on either side of the front of the chamber, one which extended periodically during autoshaping sessions (the CS + lever), and another that was always extended during autoshaping sessions (the control CS- lever). The CS+ lever also contained a white LED light embedded within it that illuminated the underside of the lever during cue presentations, and the box was equipped with a tone generator. A sucrose delivery cup was located between the levers near the floor of the front of the box. An infrared beam was incorporated into the sucrose cup to measure number of entries. A computer equipped with MED-PC software (Med Associates) controlled all events and recorded behavior during training sessions.

\section{Testing procedures: general autoshaping procedures}

Before training, rats were handled for $3 \mathrm{~d}$ and exposed to 20 sucrose pellets/rat in their home cage overnight before autoshaping training. Rats then received $1 \mathrm{~d}$ of magazine training: ad libitum sucrose pellets on a variable time (VT) $-60 \mathrm{~s}$ schedule for $20 \mathrm{~min}$ to habituate them to taking pellets from the sucrose cup. The control CS - lever was extended throughout this session, but the CS+ lever was never extended.

All autoshaping sessions consisted of 25 pavlovian pairings of the CS+ lever ( $8 \mathrm{~s}$, VT-90 s schedule) with one $45 \mathrm{mg}$ sucrose pellet UCS delivered into the sucrose cup immediately (within $0.5 \mathrm{~s}$ ) after lever retraction. Sessions ended $90 \mathrm{~s}$ after the 25 th cue (35-45 min). Rats were handled for $\sim 5$ min before each session, during which time microinjections were given, or stylets were removed, cleaned, and replaced to approximate microinjection procedures.

\section{Statistical analyses}

Autoshaping results were analyzed with mixed ANOVAs, using withinsubjects factors of drug (DAMGO vs vehicle, muscimol vs vehicle) and period (the $8 \mathrm{~s}$ before each scored cue vs the $8 \mathrm{~s}$ of each cue), and betweensubjects factor of preferred cue $\left(\mathrm{CS}+\right.$ lever or $\left.\mathrm{CS}_{\text {cup }}\right)$. Training day was also a within-subjects factor in acquisition experiments. Bonferroni corrected $t$ tests and repeated measures ANOVAs were used to assess interactions. No order effects were found between drug and vehicle days in autoshaping expression and food-intake experiments, so data were collapsed across days for all analyses. Food intake and other general behavioral effects of DAMGO and muscimol were analyzed with paired samples $t$ tests comparing drug to vehicle days. When percentage increases were reported to describe the magnitude of DAMGO and muscimol effects, raw data were adjusted by adding 1 to every score to avoid the problem of calculating percentage increases over 0 for behaviors with low baselines.

\section{Anatomical specificity of microinjection effects: Fos plumes and anatomical controls}

To map where microinjections were likely to have directly altered neuronal function adjacent to injection sites, and so assign anatomical responsibility for behavioral effects, we used a Fos plume tool in separate rats to measure local plume-shaped regions of neuronal modulation 
caused by microinjections of DAMGO, muscimol, or vehicle (total, $n=$ 17) (Peciña and Berridge, 2005; Mahler et al., 2007; Smith and Berridge, 2007). Local plumes of Fos expression usually can be distinguished from indirect elevation in more distant sites and so provide a relatively direct measure of the spread of impact on local brain tissue. To ensure maximal estimation of Fos plume spread, unconstrained by gliosis that might limit drug spread after several consecutive microinjections, Fos plumes were mapped in a separate group of naive rats under conditions equivalent to the first day of behavioral testing. Use of separate groups for Fos plume and behavioral measurements avoids underestimation of plume size, as well as induction of anticipatory Fos activation resulting from reward learning that might otherwise have imposed ceiling effects on the magnitude of drug-induced elevations in Fos. Avoiding underestimation of plume size seemed important to avoid falsely inferring localization of function beyond that which actually exists. This procedure follows similar methods to those used in previous studies (Peciña and Berridge, 2005; Mahler et al., 2007; Smith and Berridge, 2007).

Vehicle or drugs were bilaterally infused identically to above ( $\mu$ opioid agonist DAMGO, $0.1 \mu \mathrm{g}$ dose, $n=6$; $\mathrm{GABA}_{\mathrm{A}}$ agonist muscimol, $0.25 \mu \mathrm{g}$ dose, $n=3$; or sterile isotonic saline vehicle, $n=4$ ). Additional sham control rats received sham surgeries but no cannula implantation to assess spontaneous Fos levels in the absence of any cannula-associated gliosis $(n=4)$. Rats were deeply anesthetized, and 75 min later brains were removed and processed for Fos-like immunoreactivity (refer to Peciña and Berridge, 2005 for details). DAMGO and muscimol Fos plumes were mapped based on the percentage change in Fos-like immunoreactivity surrounding injection sites after DAMGO or muscimol versus controls, measured in blocks along each radial arm (Fos excitatory "plume" $=2 \times$ and $3 \times$ elevations above control levels; Fos inhibitory "anti-plume" $=>50 \%$ decline from control levels). Baselines were measured in CeA and surrounding structures in intact brains to assess normal expression, and around the site of vehicle microinjections similar to drug microinjections (Fig. 2). Adjacent slices were stained for Substance P to identify landmarks for comparison to a brain atlas (Paxinos and Watson, 2007).

Fos immunoreactivity was visualized with the avidin-biotin complex method, and images were taken of the site $5 \times$ magnification with a color camera coupled to a Leica microscope. Fos was quantified by zooming in on these images to $40 \times$ magnification to identify Fos-like immunoreactive (FLI) neurons by their shape and size (3-7 pixels at $40 \times$ magnification and 5184 pixels/square inch resolution) and counting the number of FLI cells within $0.125 \mathrm{~mm}^{2}$ block of tissue. Up to 10 consecutive blocks were counted stretching out from the microinjection center along each of seven radial arms leading away from the center of microinjection sites (a total of $\sim 80$ blocks were quantified per microinjection). To be counted as positive for Fos-like immunoreactivity, a cell was required to have a dark-black intensity that was at least $20 \%$ darker gray than background (e.g., background gray, $60 \%$ black; FLI cell, $98 \%$ black). Batch differences in background density or Fos staining were controlled by excluding any brain with levels of Fos-like immunoreactivity in a control cortical site (dorsal piriform cortex, layer 2, distant from microinjection sites) of $>2$ SDs above or below the mean number of Fos + cells per $0.125 \mathrm{~mm}^{2}$ in this structure across animals $\left[\mathrm{m}_{(\mathrm{SD})}=11.3_{(3.2)}\right]$. In addition, to ensure that drug differences in Fos were not artifacts of background contrast or nonspecific immunoreactivity between batches, at least two brains from every group were included in each batch so that drug differences could be examined within as well as across batches (uninjected, vehicle, DAMGO, and muscimol).

After Fos quantification, plume radii were measured and plotted (Peciña and Berridge, 2000, 2005; Smith and Berridge, 2005; Mahler et al., 2007; Smith and Berridge, 2007). The size of plume symbols used for mapping was based on the average radii of Fos plumes for that drug. The color of each plume symbol was coded to show the change in behavioral effects produced by drug microinjection at the corresponding site in a particular animal. The bilateral cannulae for each rat were plotted on each map to depict every placement (two sites per rat). For rats that received drug and vehicle after training, symbol color was calculated based on comparisons with each animal's own vehicle day. For rats that received microinjections during training, drug rats were plotted with plume symbols, and colors were based on comparison with the average of the vehicle group. Cannulae placements for animals receiving vehicle were marked with "Xs." Maps were always plotted separately in sagittal, coronal, and horizontal planes to construct a 3D map set.

\section{Results \\ Synopsis}

Activation of $\mu$ opioid neurotransmission in CeA by DAMGO microinjections enhanced appetitive and consummatory behaviors directed toward each rat's own prepotent CS, more than toward the alternative CS or other stimuli in the chamber. The $\mathrm{CS}+$ for sucrose reward (insertion of a metal lever through the wall into the chamber, accompanied by a tone) elicited appetitive and consummatory behaviors directed either at the CS+ lever itself, or at the metal cup where sucrose was delivered $\left(\mathrm{CS}_{\text {cup }}\right)$ within several days of training. When the CS + was presented, rats began to nibble and sniff, then bite their prepotent CS. These bouts of intense consummatory behaviors were time-locked to the $8 \mathrm{~s}$ duration of the CS+ presentation. The effect of DAMGO microinjections in CeA was to increase the number and intensity of appetitive and early-phase consummatory behaviors of the preferred CS (exploratory nibbles and sniffs; Figs. 3 and 4), often raising them to apparently frenzied levels (Fig. 3 and Video 1, available at www.jneurosci.org as supplemental material). Appetitive and consummatory behaviors directed toward the prepotent CS were reliably enhanced regardless of whether DAMGO was administered during learning or after learning. DAMGO also subsequently enhanced food intake (UCS "wanting") in the same rats, consistent with the conclusion that it enhanced the incentive salience of both food and food cues. Conversely, inactivating the CeA with muscimol during or after learning suppressed approach and consummatory behaviors directed toward the prepotent CS and suppressed UCS food intake.

\section{Classification of prepotent CS preference}

All rats developed a prepotent CS, which they approached and consumed during CS + presentations on average greater than seven times, and at least greater than three times more often than the alternative CS $\left[F_{(4,88)}=8.3, p<0.001\right.$ for group tested after learning; $F_{(2,90)}=5.4, p<0.01$ for group tested during training]. Approach and consummatory sniffing, nibbling, licking, and biting movements were directed primarily at the prepotent CS. Individual rats in the drugs post-training or drugs during training experiments preferred either the CS + lever ( $\sim 70 \%$ of rats) or the sucrose-delivering $\mathrm{CS}_{\text {cup }}$ ( $\sim 30 \%$ of rats) (Fig. 1). Over $70 \%$ of all rats preferred to approach and attempt to consume their prepotent CS nearly exclusively ( $>9$ of 10 cue interactions were with the preferred CS). Only $\sim 10 \%$ of rats interacted with both cues approximately equally (and even then slightly more with one than the other), and $20 \%$ of rats interacted with the cues on a $2: 1$ basis.

Phasic peaks of consummatory behavior toward the prepotent CS were always time-locked to insertions of the CS+ lever through the wall into the chamber $\left[F_{(1,22)}=78.6, p<0.001\right]$, lasting the duration of the $8 \mathrm{~s} \mathrm{CS}+$, and decaying immediately or within seconds of the lever's disappearance (even when the consummatory behavior was directed to the $\mathrm{CS}_{\text {cup }}$ that remained present afterward). Each rat's preference for a particular CS stimulus remained stable across days, and CS prepotency was not redirected by DAMGO but merely intensified for the already prepotent CS [ (and not changed for the other CS); $\chi^{2}=1.8$, n.s.; no interaction of type $\times$ drug on CS + lever nibbles and sniffs, $F_{(2,90)}$ $=2.4$, n.s.)]. 

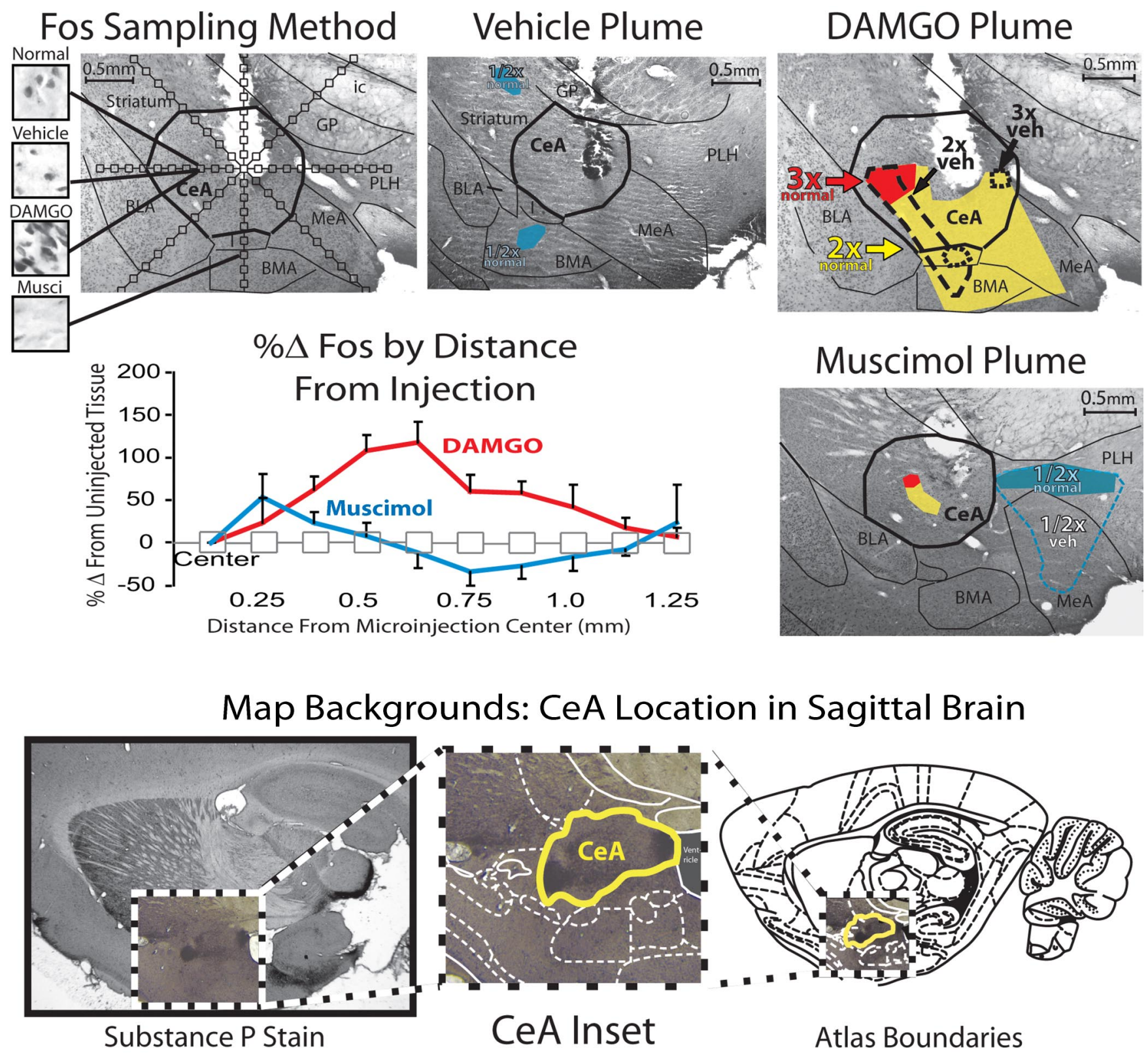

Figure 2. Fos plumes in amygdala. Fos Sampling Method, Illustrates the method used to sample Fos expression around injection sites, in which radial arms for sampling extend from the center of microinjection sites, viewed in a coronal plane. Fos-expressing neurons are counted in $62.5 \times 62.5 \mu \mathrm{m}$ blocks on arms spaced at $62.5 \mu \mathrm{m}$ intervals; $5 \times$ magnification. Insets show sample tissue blocks from equivalent sites in brains injected with nothing, vehicle alone, DAMG0, or muscimol; photo taken at 40X magnification with exposure time adjusted to clearly show Fos expression. Vehicle plume: Example changes in Fos following vehicle microinjection alone, compared with uninjected tissue. DAMG0 and muscimol plumes, Example DAMG0 and muscimol-induced plumes of Fos modulation. Colors indicate areas of Fos elevation by $2 \times$ (yellow) or $3 \times($ red) over uninjected control levels for the relevant structure. Lines represent areas of Fos elevation by $2 \times$ (dashed line) or $3 \times$ (dotted line) over vehicle microinjection levels at equivalent sampling boxes and brain areas. Regions of Fos inhibition caused by muscimol began on average $0.36 \mathrm{~mm}$ from microinjection centers. \% $\Delta$ Fos by distance from injection: Fos activation was plotted as percentage change from uninjected tissue levels, as a function of distance from the center of microinjection. Data were averaged across rats for the vertical sampling arm, extending ventrally from the injection site and compared with uninjected control Fos levels in equivalent brain areas. Map backgrounds - CeA anatomical stain: Anatomical borders of the CeA were visualized in a sagittal view with a Substance P stain (left), and the inset shows the background used for mapping Figures $4-6$ within the larger brain and atlas boundaries [right; adapted from Paxinos and Watson (2007), maximum rostrocaudal and dorsoventral extent of CeA depicted]. Injection sites in behavioral and Fos animals were identified in coronal sections with adjacent slices stained for Substance $P$ to identify the CeA, then transferred to a sagittal view for presentation. CeA, central amygdala; BLA, basolateral amygdala; MeA, medial amygdala; BMA, basomedial amygdala; I, intercalated nuclei; ic, internal capsule; GP, Globus pallidus; PLH, posterior lateral hypothalamus.

CeA DAMGO enhances incentive salience of previously learned prepotent CSs

DAMGO microinjections specifically enhanced by up to $220 \%$ over vehicle levels the number of nibbles and sniffs directed toward a rat's prepotent CS that it had previously acquired during several undrugged days of training $\left[\right.$ veh $\mathrm{m}_{(\mathrm{SEM})}=3.8_{(0.4)}$; DAMGO $\left.\mathrm{m}=5.0_{(0.4)} ; F_{(1,21)}=9.2, p<0.01\right]$. DAMGO increased the number of appetitive and consummatory behaviors directed toward the preferred CS only and not toward the nonpreferred CS [interaction of cue type $\times$ drug on during cue nibbles and sniffs, $\left.F_{(1,21)}=6.7, p<0.05\right]$.

Thus, in CS + lever preferring rats, DAMGO specifically enhanced consummatory nibbles and sniffs of the CS+ lever by up to $218 \%$ of vehicle day levels [mean increase $=146 \%$, veh $\mathrm{m}=$ 


\section{CS+ Triggered Consummatory Behaviors}
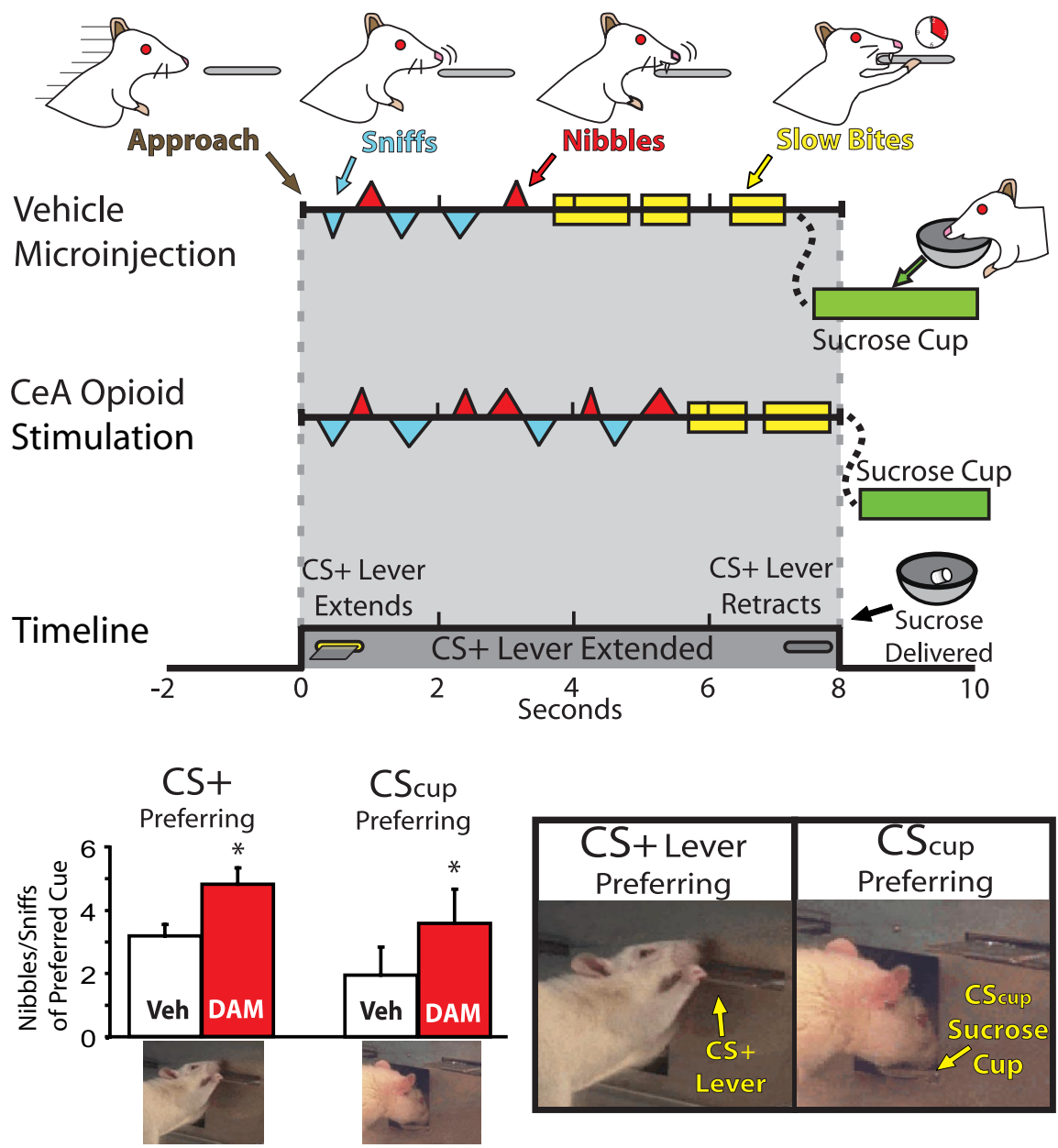

Figure 3. Effects of DAMGO on the microstructure of CS appetitive- consummatory behavior. The behavior of a typical rat with CeA cannulae following vehicle and DAMGO microinjections on separate days following acquisition of preference for the $\mathrm{CS}+$ lever is shown in the top section. After vehicle or no microinjections, CS + lever-prepotent rats typically approach, nibble, and sniff the CS + lever when it extends, then transition to a period of slower, discrete bites. When DAMG0 is administered in CeA, the nibbling and sniffing period is extended at the expense of the slow biting period. Bar graphs at the bottom show that anterior CeA DAMGO enhances consummatory behaviors directed at the $C S+$ lever or $C S_{\text {cup }}$ over vehicle levels in $C S+$ lever or $C S_{\text {cup }}$-preferring rats, respectively. Sucrose pellet is delivered $8 \mathrm{~s}$ after lever extension, coincident with lever retraction. See video 1 , available at www.jneurosci.org as supplemental material, for examples of $C S+$ lever and $C S_{\text {cup }}$ consummatory behaviors. * indicates difference from vehicle for all CeA animals, $p<0.05$.

$3.4_{(0.3)}$, DAMGO m $\left.=4.9_{(0.5)} ; t_{(14)}=4.7, p<0.001\right]$ while not affecting these behaviors directed toward the nonpreferred $\mathrm{CS}_{\text {cup }}$ [veh $\mathrm{m}=0.3_{(0.1)}$, DAMGO m $=0.4_{(0.2)} ; t_{(14)}=1$, n.s.]. Conversely in $\mathrm{CS}_{\text {cup }}$-preferring rats, DAMGO microinjections in anterior CeA increased nibbles and sniffs of the sucrose cup (and entries into it) to up to $200 \%$ of vehicle levels when the CS+ was present [mean increase nibbles/sniffs $=120 \%$, veh $\mathrm{m}_{(\mathrm{SEM})}=$ $2.56_{(0.8)}$, DAMGO m $=3.95_{(0.9)} ; t_{(3)}=10.1, p<0.01$; entries $t_{(3)}$ $=7.3, p<0.01]$, but did not affect nibbling and sniffing interactions with the $\mathrm{CS}+$ lever $\left[\mathrm{veh} \mathrm{m}=0.6_{(0.6)}\right.$, DAMGO m $=0.5_{(0.2)} ; t_{(3)}$ $=0.9$, n.s. $]$.

DAMGO also intensified the rate (number per second) of preferred CS nibbling and sniffing, suggesting that the increased quantity of these behaviors reflects enhanced vigor or intensity of investigatory nibbling and sniffing (per unit of time) and not just a prolongation of the period spent showing the behaviors [DAMGO effect on preferred but not nonpreferred cue nibbling/ sniffing rate, $F_{(1,21)}=12.0, p<0.01$; preferred cue, $t_{(21)}=4.2, p<0.001$; nonpreferred, $t_{(21)}=1.5$, n.s.]. As DAMGO enhanced appetitive/consummatory nibbles and sniffs that typically initiated bouts of ingestive-type behaviors, it simultaneously excluded other more instrumental and terminal behaviors in a manner suggesting response competition, thus reducing computer-scored lever presses to only $47 \%$ of vehicle $\left[F_{(1,14)}=14.8, p<0.01\right]$ and reducing slower, discrete bites (lasting $>0.5 \mathrm{~s}$ each in duration) that otherwise tended to terminate ingestive sequences [79\% of vehicle day, $F_{(1,14)}=12.4, p<$ $0.05]$.

CeA DAMGO during training also enhanced CS consummatory behavior Similarly, the separate group of rats that received opioid stimulation of $\mathrm{CeA}$ while they were initially learning CS/UCS associations (days 1-6) showed enhancements of appetitive and consummatory behaviors directed toward their prepotent CS, but only beginning around day 4 [days 4-6: veh $\mathrm{m}_{(\mathrm{SEM})}=3.2_{(0.5)}$, DAMGO m = $5.0_{(0.4)} ; F_{(1,47)}=7.9, p<0.01$ ] (Fig. 4; supplemental Fig. 2, available at www.jneurosci. org as supplemental material). On the initial three training days, DAMGO did not significantly increase cue nibbles or sniffs during CS+ presentations $\left[F_{(1,47)}=2.6\right.$, n.s.], nor did it advance the day of preference acquisition $\left[t_{(47)}=0.26\right.$, n.s. $]$, although it did increase sucrose cup entries in all animals during non-cue periods [main effect of drug in precue period for days $\left.1-3, F_{(1,47)}=8.6, p<0.01\right]$.

\section{Detailed behavioral topography of $C e A$ DAMGO effect}

Consummatory behaviors directed toward preferred CSs were usually observed to follow a predictable sequence, which was similar to behaviors observed in UCS food intake. When eating a UCS chow pellet, rats approach and vigorously nibble and sniff it for 2-10 s, then pick up the pellet and subsequently transition into slower, more discrete bites that terminate the consummatory sequence with actual ingestion of food. Food cueoriented behaviors in autoshaping followed a similar sequence, with two to three intense, frenzied bouts of sniffs and nibbles, which are repeated several times in rough alternation during each 1-2 s bout. Thus, nibbles and sniffs appear to be a transitional appetitive-consummatory phase of behavior, reflecting initial moments of intense exploration and motivated interest in the CS, similar to what would be expected of an attractive, salient stimulus. Sniffs and nibbles were usually followed by a period of slower, discrete bites lasting $\sim 0.5-1 \mathrm{~s}$ each, similar to the terminal stereotyped biting and swallowing movements of ingesting actual food. Slow bites usually terminated the CS consummatory sequence and competed with the preliminary period of nibbling and sniffing for expression within $8 \mathrm{~s}$ cue periods. For prepotent 


\section{$\mathrm{CeA} \mu$ Opioid Stimulation After Training $\quad \mathrm{CeA} \mu$ Opioid Stimulation During Training}

\section{Preferred CS}
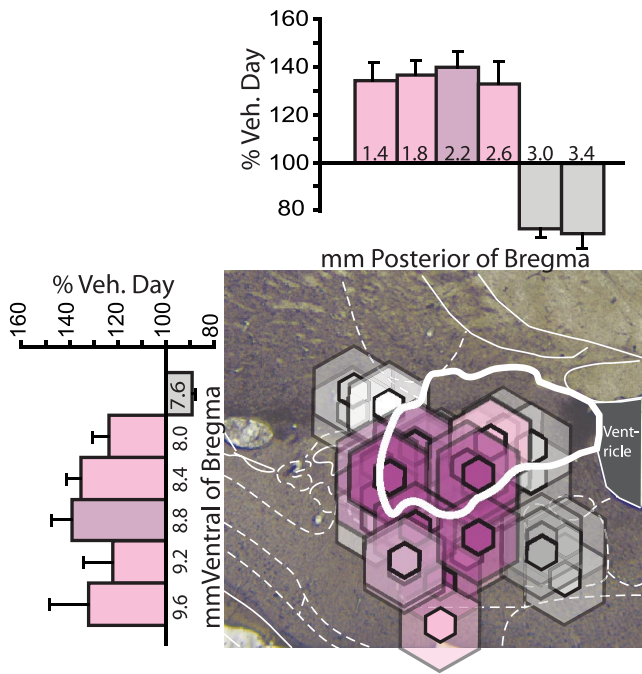

\section{Non-Preferred CS}

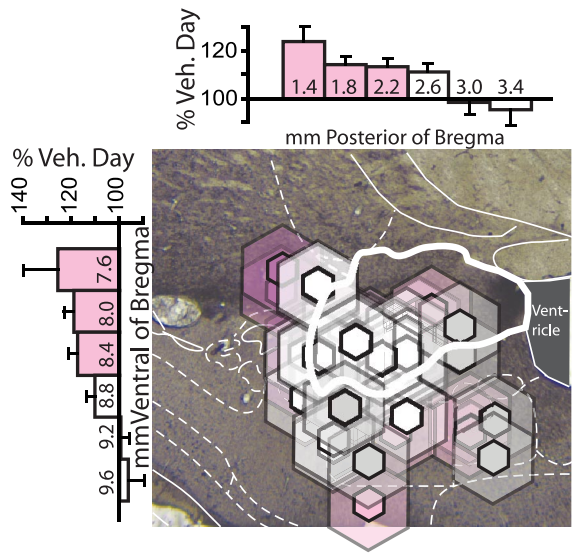

All CeA Animals

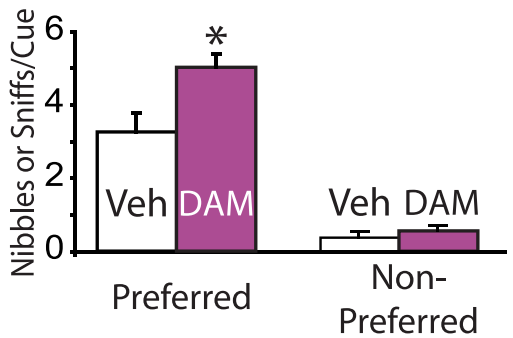

\section{Preferred CS}
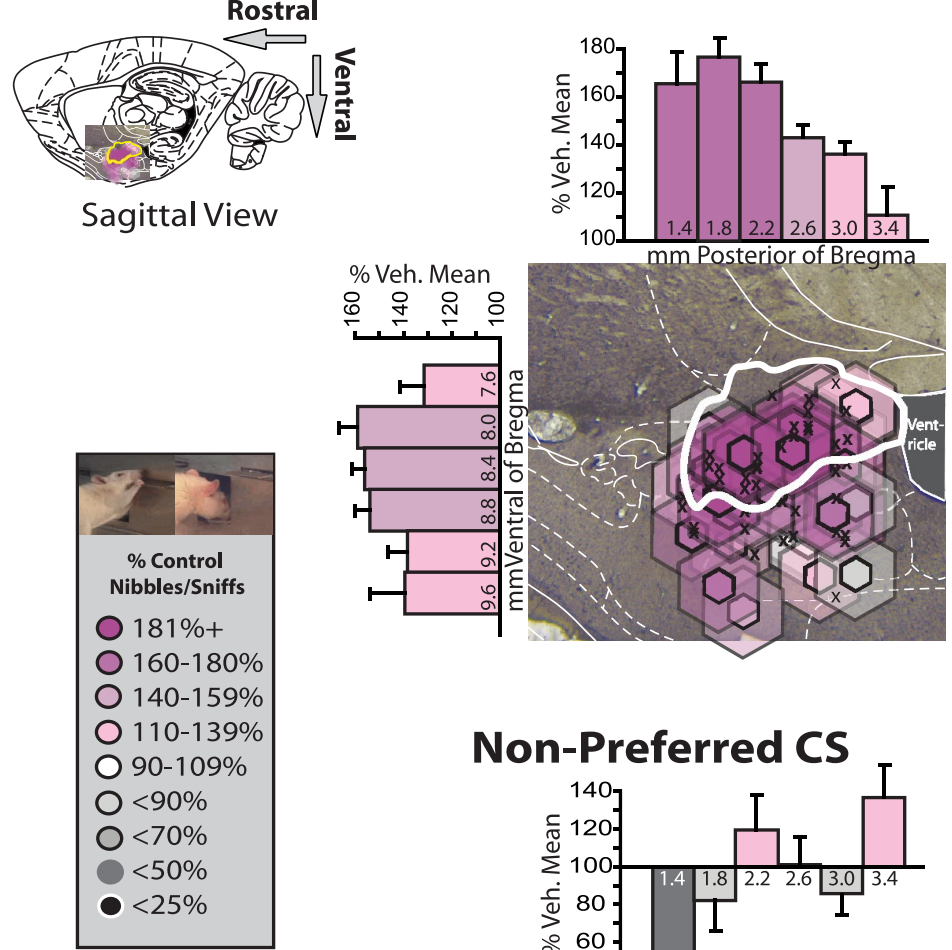

\section{Non-Preferred CS}

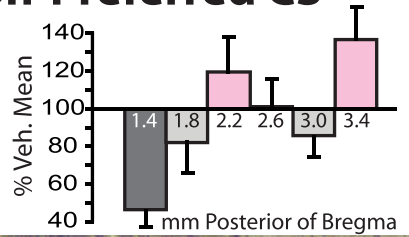

$\%$ Veh. Mean

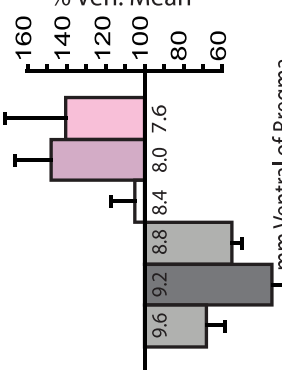

All CeA Animals

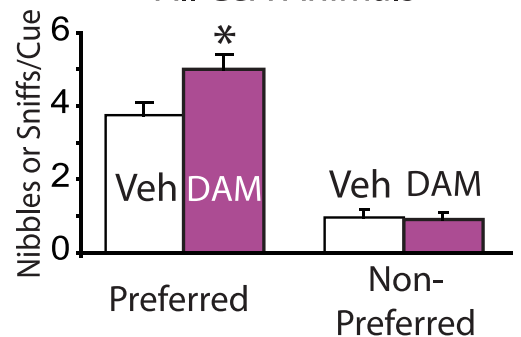

Figure 4. Stimulating CeA opioids after or during autoshaping training specifically stimulates nibbles and sniffs of the prepotent CS. CeA DAMGO enhancement of "wanting" for preferred and nonpreferred cues are mapped rat-by-rat based on the intensity of DAMG0 effects at the mapped microinjection sites (color) and average DAMGO Fos plumes measured at similar sites (size). CeA $\mu$-opioid stimulation after training (left column): after animals learned to preferentially approach and interact with one of the two CSs, DAMGO enhanced nibbles and sniffs only of the prepotent CS, but not the alternative CS during cues. CeA $\mu$-opioid stimulation during training (right column): when DAMG0 was administered while animals were learning the autoshaping task; again it enhanced CS consummatory behavior selectively toward the preferred CS, once the preference became established (average cue nibbles and sniffs for the last 3 training days shown). Hexagonal symbol colors denote DAMGO modulation of prepotent CS nibbles and sniffs, calculated as percentage change from vehicle day in the same animals, or the mean of the vehicle animals in the DAMGO during training experiment. Inner symbols represent average diameter of $3 \times$ Fos enhancement over uninjected tissue levels, surrounded by semitransparent halos that show $2 \times$ Fos enhancement zones. ' $X$ ' symbols indicate cannulae placements for rats receiving vehicle microinjections in the between-subjects design, DAMG0-during-training experiment. Bars along rostrocaudal and dorsoventral axes show the intensity of DAMGO effects (mean \pm SEM percentage of vehicle levels) within each $0.4 \mathrm{~mm}$ wide level, centered on the labeled coordinate; a plume symbol can contribute to more than one bar when it straddles multiple levels). At bottom, summary data for all animals with cannulae placements in CeA are shown for DAMG0 administered after training (left) and DAMG0 during training (right). Bar colors in all cases reflect mean percentage change from vehicle in that zone. * indicates difference from vehicle for all CeA animals, $p<0.05$. 
CSs, the main effect of DAMGO in CeA was to prolong the initial phase of intense exploratory nibbling and sniffing, postponing or even displacing the terminal bites because the lever disappeared before the rat stopped showing nibbles and sniffs (Fig. 3). DAMGO specifically potentiated this initial phase of vigorous nibbles and sniffs, as if the CS + lever had suddenly become a more attractive and salient stimulus worthy of intense, vigorous investigation. In short, DAMGO did not simply enhance all preexisting cue-directed behaviors, or cause rats to confuse the CS with food or actually try to eat the metal object (with terminal bites), but specifically potentiated a pattern of appetitive approach and intense initial consummatory behaviors that plausibly reflect incentive salience.

\section{Specificity of CeA DAMGO effects}

DAMGO selectively enhanced appetitive and consummatory behaviors that were triggered by appearance of the CS + lever, and did not enhance cue-directed nibbles and sniffs in absence of the $\mathrm{CS}+$ lever. Even for the $\mathrm{CS}_{\text {cup }}$ that was always present, DAMGO failed to enhance approach or consummatory behaviors in the absence of the CS + lever [interaction of cue period $\times$ drug on preferred cue nibbles and sniffs, $\left.F_{(1,21)}=9.0, p<0.01\right]$. Thus, for all CS consummatory behaviors, enhancements were timelocked to the presence of the $\mathrm{CS}+$, occurring as phasic peaks that typically decayed to baseline within 1-2 s of the end of CS+ presentation.

DAMGO enhancements of CS consummatory behaviors reversed and disappeared when rats were tested drug-free on a subsequent day (48-96 h after DAMGO; day 8 of training, after DAMGO administration on day 6 or 7 ). On the postdrug retest day, nibbles and sniffs were reduced from their DAMGOinduced peak in the same rats [retest $\mathrm{m}_{(\mathrm{SEM})}=3.8_{(0.3)}$ vs DAMGO $\left.\mathrm{m}=5.0_{(0.4)} ; F_{(1,21)}=8.1, p=0.01\right]$ and were no longer different from the initial vehicle day $\left[\mathrm{m}=3.8_{(0.4)} ; F_{(1,21)}=0.1\right.$, n.s.]. Therefore, DAMGO incentive salience enhancements were not permanent but reflected a temporary motivation change related to current opioid levels in CeA.

CeA opioid enhancements were highly focused, and specific to a rat's particular prepotent CS, and did not extend to either the CS - control lever or to the non-prepotent CS [no post-training drug effect on CS - nibbles and sniffs, $t_{(23)}=1.4$, n.s., or presses, $t_{(23)}=1.0$, n.s.; similarly no pretraining drug effect on CS - lever nibbles/sniffs, $F_{(1,27)}=0.3$, n.s.; or presses, $F_{(1,27)}=0.1$, n.s.]. As mentioned above, only a few rats approached and attempted to consume the CS+ lever and $\mathrm{CS}_{\text {cup }}$ cues approximately equally during CS + presentations. Even for these rats $(n=3)$, DAMGO selectively enhanced approach and attempted consumption of only one cue and never both (CS+ lever for two rats, and $\mathrm{CS}_{\text {cup }}$ for the remaining rat). Thus, even in the few rats that are ordinarily attracted to both CSs, CeA DAMGO enhanced the incentive salience of only one CS at a time.

\section{Baseline effect of CeA DAMGO in the absence of cues}

DAMGO appeared to produce a general locomotor enhancement in the absence of the CS + . In the periods between CS+ presentations, DAMGO increased the number of head entries into the sucrose cup for both $\mathrm{CS}+$ lever and $\mathrm{CS}_{\text {cup }}$ preferring rats [main effect of drug in precue period, $F_{(1,22)}=331.6, p<0.001$ ], especially CS + lever preferring rats whose preferred CS was not then present [interaction of rat cue preference $\times$ drug, $F_{(1,22)}=6.0$, $p<0.05]$. Touches of the control CS - lever, as well as the number of rears were also increased by DAMGO during between-cue periods [interaction of drug $\times$ period, CS - lever, $F_{(1,22)}=6.0$, $p<0.05$; rears, $\left.F_{(1,22)}=13.8, p=0.001\right]$.

\section{CeA DAMGO enhances food UCS consumption}

In separate food-intake tests, opioid stimulation of CeA doubled UCS chow intake compared with vehicle levels [grams eaten, $t_{(23)}$ $=6.1, p<0.001]$. DAMGO also increased the cumulative duration of eating behavior and number of eating bout initiations to $>350 \%$ of vehicle levels [duration, $t_{(23)}=2.9, p<0.01$; initiations, $t_{(23)}=3.1, p<0.01$ ] (see Fig. 6). DAMGO also increased the number of sniffs of food pellets [ $165 \%$ increase in frequency; $\left.t_{(23)}=2.4, p<0.05\right]$, and pellet pickups [234\% frequency increase; $t_{(23)}=2.1, p=0.05$ ], although the total number of sniffs displayed toward food per bout was always far lower than was directed by the same rats toward their prepotent CS in autoshaping testing (0.5-2 s of UCS sniffing; $4-7$ s prepotent CS sniffing and nibbling). In contrast, DAMGO did not alter drinking behavior (drinking bouts, drinking time, drinking time/bout: $t$ test values $<1$, n.s.; supplemental Fig. 1, available at www.jneurosci. org as supplemental material) or chewing of non-food objects in the cage, such as control lever or lights, pieces of bedding or excrement $(t$ test values $<1$, n.s.).

\section{Muscimol inactivation of CeA suppresses incentive salience of prepotent CSs}

Inactivation of the CeA by muscimol microinjection dramatically suppressed cue-triggered consummatory behaviors directed at the prepotent CS, reducing levels to $10-30 \%$ of vehicle levels [vehicle $\mathrm{m}_{(\mathrm{SEM})}=4.1_{(0.3)}$; muscimol $\mathrm{m}=0.9_{(0.3)} ; t_{(21)}=3.3, p<$ 0.01]. Muscimol did not affect the already low levels of consummatory behaviors directed toward the nonpreferred CS [prepotent vs nonpreferred cue, $F_{(1,21)}=64.7, p<0.001$; no effect of muscimol on nonpreferred cue, vehicle $\mathrm{m}=0.6_{(0.2)}$, muscimol $\mathrm{m}=0.4_{(0.2)} ; t_{(21)}=2.0$, n.s.] (Fig. 5). Muscimol microinjection reduced cue-triggered consummatory nibbles and sniffs only of the preferred CS in both CS+ lever and $\mathrm{CS}_{\text {cup }}$ preferring rats [cue period $\times$ drug interaction on nibbles and sniffs CS + lever rats, vehicle $\mathrm{m}=3.4_{(0.4)}$, muscimol $\mathrm{m}=0.5_{(0.3)} ; F_{(1,22)}=25.3, p<$ $0.001 ; \mathrm{CS}_{\text {cup }}$ rats, vehicle $\mathrm{m}=3.1_{(0.5)}$, muscimol $\mathrm{m}=0.7_{(0.4)}$; $\left.F_{(1,22)}=12.12, p<0.001\right]$, as well as CS + lever slow bites in CS + lever rats (period $\times$ drug interaction for slow bites, vehicle $\mathrm{m}=$ $0.7_{(0.3)}$, muscimol m $\left.=0.2_{(0.1)} ; F_{(1,14)}=9.0, p=0.01\right]$. Muscimol did not significantly affect the low level of CS - control lever pressing during cue periods but did marginally reduce CSpressing in the absence of the CS $+\left[F_{(1,21)}=4.9, p<0.5\right.$; during cue, $t_{(21)}=1.4$, n.s.; precue period, $t_{(21)}=1.8, p=0.9$ ].

Muscimol similarly reduced prepotent CS approach and consummatory interactions when administered during training, with lever consummatory behaviors being decreased by $>70 \%$ from vehicle levels by day 4 [interaction of drug $\times$ test day for $\mathrm{CS}+$ lever nibbles and sniffs, $F_{(3,18)}=4.3, p<0.05, t$ test on day $\left.4, t_{(6)}=3.5, p<0.05\right]$. However, rats still developed cuetriggered approach responses to the sucrose cup during cue periods [main effect of pre-cue period vs during cue period, $F_{(1,6)}=$ $7.3, p<0.05$ ] and did not significantly differ from vehicle rats in $\mathrm{CS}_{\text {cup }}$ entries [vehicle $\mathrm{m}=0.84_{(0.25)}$, muscimol $\mathrm{m}=0.77_{(0.34)}$, $F_{(1,6)}=0.01$, n.s.; supplemental Fig. 3, available at www. jneurosci.org as supplemental material]. Thus overall, inactivation of CeA prevented rats from attributing strong incentive salience to a discrete $\mathrm{CS}+$, whether they were still learning its motivational significance during training or had already learned to target incentive salience upon it during previous training.

Suppression of CS appetitive-consummatory behaviors by 


\section{CeA Inactivated After Training}
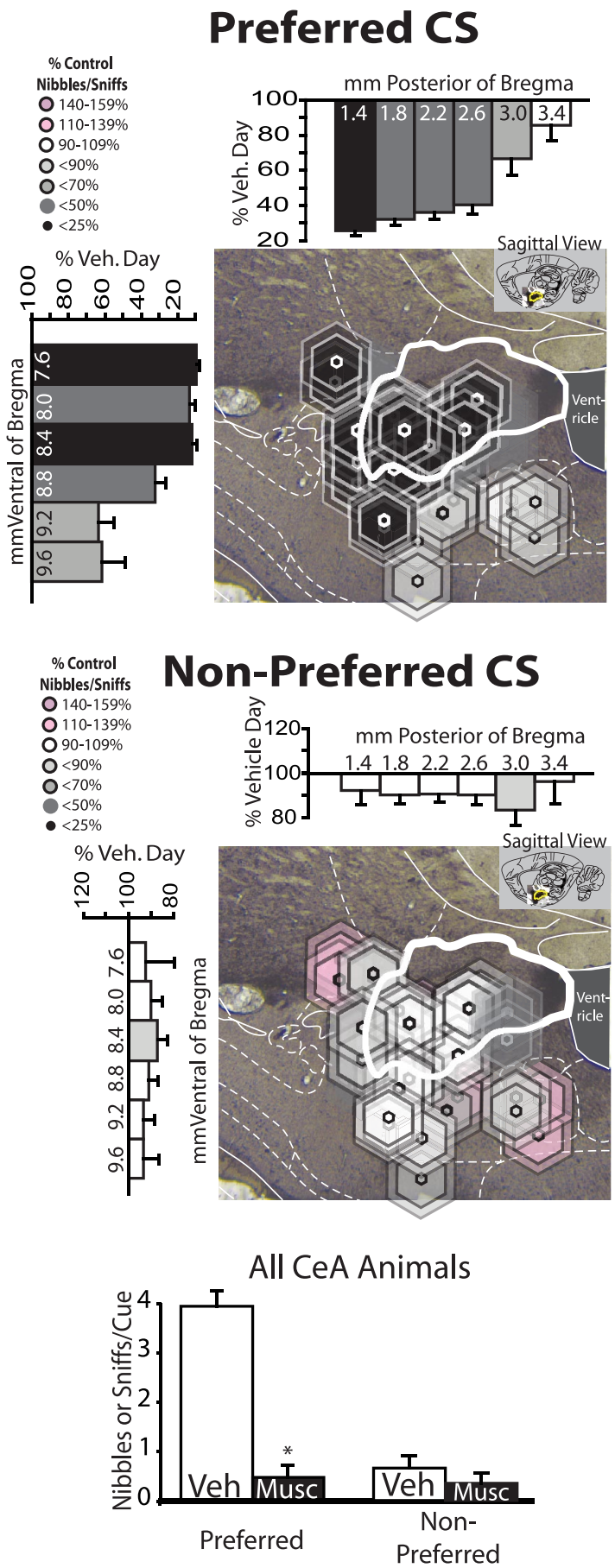

Figure 5. Muscimol inactivation of CeA after autoshaping training reduces nibbles and sniffs of the prepotent $C S$. Inactivating the CeA with muscimol after training (percentage change from vehicle day in the same rats) dramatically reduced prepotent cue nibbling and sniffing but does not consistently affect these appetitive- consummatory interactions with the nonpreferred CS. Summary data for all animals with cannulae placements in CeA are shown at bottom. ${ }^{*}$ indicates difference from vehicle for all CeA animals, $p<0.05$.

muscimol appeared to be stronger at anterior sites in CeA (defined as between 1.4 and $2.04 \mathrm{~mm}$ behind bregma, 31\% of vehicle levels) than at posterior sites [2.04-3.4 mm behind bregma, 46\% of vehicle; main effect of cannula placement on prepotent CS nibbles and sniffs $\left.\left(F_{(1,13)}=9.5, p<0.01\right)\right]$. The rostrocaudal difference suggested that anterior CeA may be more sensitive than posterior CeA for modulating the incentive salience of prepotent CSs. Muscimol inactivation of amygdala suppressed preferred cue consummatory interactions whether injections were bilaterally contained within CeA or only unilaterally contained [bilateral CeA, vehicle $\mathrm{m}_{(\mathrm{SEM})}=4.0_{(0.5)}$; muscimol $\mathrm{m}=0.5_{(0.4)}$; $t_{(8)}=7.0, p<0.001$; unilateral CeA, vehicle $\mathrm{m}=3.1_{(0.7)}$; musci$\left.\mathrm{mol} \mathrm{m}=0.6_{(0.4)} ; t_{(5)}=3.7, p<0.05\right]$. Unlike DAMGO, musci$\mathrm{mol}$ also suppressed consummatory interactions with the prepotent CS even when injections were centered outside the CeA borders (e.g., in BMA or BLA).

In baseline periods between CS + presentations, muscimol decreased corner sniffing and rearing behavior [interaction of period $\times$ drug; sniffing, $F_{(1,22)}=23.6, p<0.001$; rears, $F_{(1,22)}=$ $19.3, p<0.001]$ and conversely increased the amount of time rats spent totally immobile $\left[F_{(1,22)}=8.4, p<0.01\right]$.

\section{CeA muscimol reduces UCS food and water intake}

During autoshaping test sessions, muscimol decreased eating behavior and suppressed the retrieval and consumption of UCS sucrose pellets to $30-40 \%$ of vehicle levels $\left[t_{(8)}=7.8, p<0.001\right.$ for post-learning test; $F_{(1,6)}=13.31, p<0.01$ for during-training test; however, sucrose pellet eating rebounded to $>85 \%$ from the second test day on in the during-training DAMGO experiment].

In the separate free-intake test, using normal chow pellets that may have been less palatable than sucrose, muscimol microinjections in CeA completely abolished food intake to zero $\left[t_{(8)}=2.8\right.$, $p<0.01]$. Muscimol also reduced video-scored eating behaviors, including the number of eating bouts $\left[t_{(8)}=3.5, p=0.001\right]$, cumulative duration of time spent eating $\left[t_{(8)}=3.0, p<0.01\right]$ (Fig. 6), and the total number of food sniffs [ $51 \%$ of vehicle, $t_{(8)}=$ $2.5, p<0.05]$. CeA muscimol also reduced water intake bouts $\left[t_{(8)}=4.2, p<0.001\right]$ and time spent drinking $\left[t_{(8)}=4.0, p<\right.$ 0.001 ; supplemental Fig. 1, available at www.jneurosci.org as supplemental material] but did not affect the number of bouts or total time spent chewing objects such as bedding or excrement $(t$ test values $<1$, n.s.).

We note that functional inactivation by muscimol here was intended to assess generally whether CeA participation was necessary to phasically want a CS for reward by inhibiting all CeA neurons with $\mathrm{GABA}_{\mathrm{A}}$ receptors, regardless of whether they also possessed opioid receptors. To know whether endogenous opioid signals in CeA in particular mediate normal "wanting" of a cue, future experiments could use microinjections of an opioid antagonist into CeA. Consistent with this possibility, we note that CeA microinjections of the opioid antagonist naltrexone do at least suppress UCS food intake (Giraudo et al., 1998; Glass et al., 2000).

\section{Other general behaviors elicited by amygdala opioid stimulation or muscimol inactivation}

Defensive treading behavior elicited by DAMGO and muscimol Beyond reward-related behavior, the CeA participates in fearrelated behavior (Paré et al., 2004; Zimmerman et al., 2007), and our CeA manipulations may have additionally modulated defensive motivation. Defensive treading is an innate fearful response in rodents to aversive stimuli that can be actively managed, elicited in the wild by rattlesnakes, scorpions, or similar threats and elicited in the lab by discrete threats like avoidable shock prods (Owings and Coss, 1977; Treit et al., 1981; Rodgers et al., 1997). Treading involves forward motions of the forepaws that push and throw dirt or bedding forcefully in the direction of the perceived threat (predator, laboratory shock prod in chamber, or experi- 


\section{CeA DAMGO Enhances Time Eating}
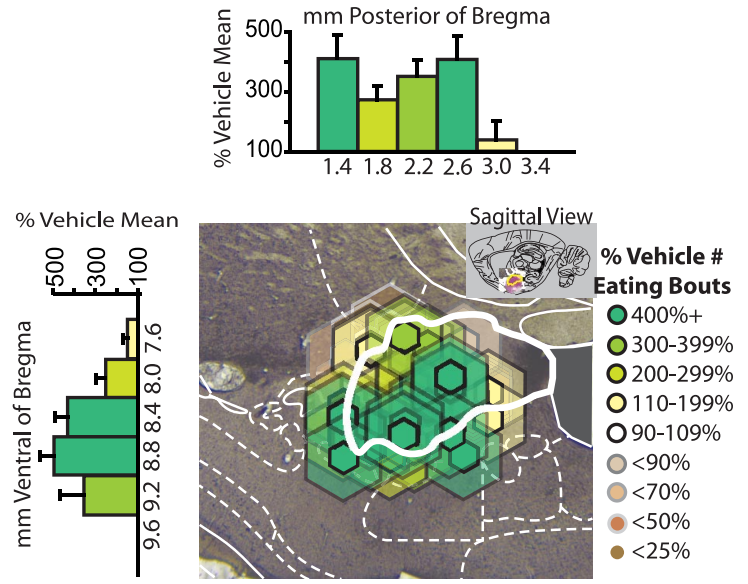

\section{CeA Muscimol Reduces Time Eating}

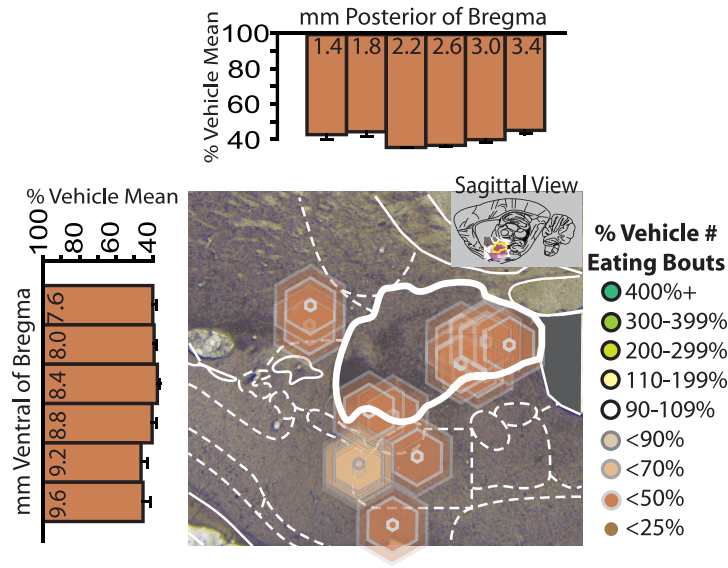

\section{Summary of CeA Animals}

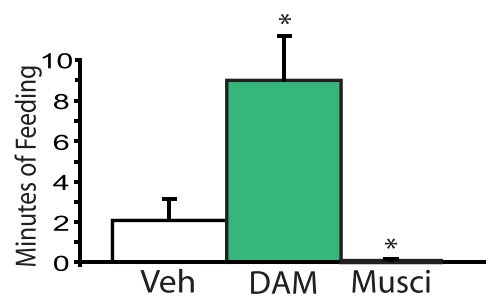

Figure 6. Opposite effects of CeA opioid stimulation and muscimol inactivation on food intake. DAMGO enhances and muscimol reduces the time spent eating in a $1 \mathrm{~h}$ period after microinjections into the CeA and immediate vicinity. Summary data for all animals with cannulae placements in CeA are shown at bottom. * indicates difference from vehicle for all CeA animals, $p<0.05$.

menters nearby), and microinjections of GABA/glutamate agents in limbic brain structures can sometimes elicit treading behavior in the absence of a normally threatening stimulus (Reynolds and Berridge, 2001; Smith and Berridge, 2005).

Defensive paw treading behavior was observed in $\sim 20 \%$ of rats after CeA DAMGO in chambers containing corncob bedding, but only very rarely observed after vehicle microinjections $[<4 \%$ of rats tread more than once/ $1 \mathrm{~h}$ session; main effect of drug, $F_{(1,22)}=5.9, p<0.05$; supplemental Fig. 4, available at www.jneurosci.org as supplemental material]. DAMGO- induced treading was moderate in intensity $\left[\mathrm{m}=4.5_{(2.5)} \mathrm{s} / \mathrm{h}\right]$ and was oriented toward the corners and walls of the testing cage, especially the front wall through which the experimenters and cameras could be seen. Defensive treading generally alternated with periods of food intake in chambers that contained both chow and bedding stimuli and was never observed in autoshaping chambers that had bare floors. Rats were sometimes even observed to sniff and pick up a piece of food, quickly drop it and tread at it for 1-2 s, then immediately return to the food pellet and begin eating again. DAMGO-induced treading occurred most frequently in animals with placements centered in the ventral half of the CeA, bordering on BLA and BMA (8.4-9.8 mm ventral of bregma) compared with rats with dorsal CeA sites [7.0-8.4 mm ventral of bregma; drug $\times$ dorsal/ventral placement interaction, $F_{(1,22)}=4.3, p=0.05$; supplemental Fig. 4 , available at www. jneurosci.org as supplemental material).

Inhibition of CeA by muscimol microinjections elicited stronger defensive treading behavior than CeA DAMGO $[\mathrm{m}=$ $34.6_{(12.8)}$ seconds treading/session; $71 \%$ of animals; muscimol vs vehicle, $t_{(8)}=2.7, p<0.05$; supplemental Figure 4, available at www.jneurosci.org as supplemental material], at levels comparable to that seen after muscimol or AMPA antagonist microinjections in the caudal accumbens shell (Reynolds and Berridge, 2008). Muscimol-induced treading was more prolonged and did not alternate with periods of food intake as with DAMGOinduced treading, and was very rarely oriented toward food pellets (and again only occurred in testing chambers that contained bedding).

Other behavioral effects of DAMGO and muscimol

DAMGO moderately enhanced general locomotion in foodintake chambers, including cage crosses $\left[147 \%\right.$ of vehicle, $t_{(8)}=$ 2.2, $p<0.05$ ] and rears [146\% vehicle, $t_{(8)}=2.2, p<0.05$ ]. However, DAMGO decreased the number of grooming bouts $\left[t_{(8)}=2.4, p<0.05\right]$ and did not affect the amount of time spent sleeping $\left[t_{(8)}=1.3\right.$, n.s. $]$.

Muscimol generally decreased locomotion, including rearing [54\% of vehicle, $t_{(8)}=2.6, p=0.01$ ] and decreased grooming [17\% of vehicle levels, $\left.t_{(8)}=3.7, p=0.001\right]$.

Most rats $(83 \%)$ also displayed unusual spontaneous digging movements after muscimol in CeA in both autoshaping and food-intake testing situations (Baldo et al., 2005), punctuated by brief (5-30 s) periods of total immobility, which were never seen after vehicle or DAMGO microinjections [main effect of muscimol on digging in autoshaping boxes, $F_{(1,22)}=20.4, p<0.001 ; t$ test on muscimol vs vehicle in food intake chambers, $t_{(8)}=2.4$, $p=0.05$; supplemental Fig. 1, available at www.jneurosci.org as supplemental material]. These were different from defensive treading in movement morphology (digging scooped or pulled bedding toward the body, treading pushed bedding away), length of bouts (digging occurred in bouts of 10-240 s, treading occurred in bouts of $\sim 1 \mathrm{~s}$ ), orientation (treading was directed at cage corners or other discrete stimuli, digging was not directed at any stimulus), and ongoing behavior (digging occurred during linear or circular forward locomotion, while treading occurred as the animal was stationary or moving backward).

\section{Fos plumes and functional spread of drug microinjections}

Fos plume maps were constructed from histology performed on brains of separate rats, which indicated that the local regions of direct modulation produced by the drug/dose/volume parameters of microinjections at placement sites comparable to those used in behavioral experiments above were mostly contained 
within the CeA nucleus. Fos was measured by counting the number of FLI neurons in $\sim 80$ sample boxes $(0.125 \times 0.125 \mathrm{~mm} ; 10$ boxes along each of eight radial arms, spaced at $0.125 \mathrm{~mm}$ increments along each arm). Uninjected control rats spontaneously expressed an average of approximately four to five Fos-positive neurons per $0.125 \mathrm{~mm}^{2}$ block in central, basomedial, and medial nuclei of amygdala $\left[\mathrm{CeA} \mathrm{m} \mathrm{(SEM}_{2}=4.19_{(0.2)}\right.$; BMA m $=4.15_{(0.3)}$, MeA $\left.\mathrm{m}=4.5_{(0.5)}\right]$, and this baseline number was not significantly altered by vehicle microinjections $\left[\mathrm{m}=4.8_{(0.5)}\right.$ cells per sampling block]. DAMGO more than tripled Fos expression from 4 to 12 neurons within the inner portion of a plume-shaped zone that stretched ventrally, medially, and/or laterally from the microinjection center $\left[\mathrm{m}=12.6_{(1.3)}\right.$ Fos-positive cells $/ 0.125 \mathrm{~mm}^{2}$ sampling box]. In an outer zone extending further from the injection center, DAMGO more than doubled the number of Fos-positive cells over control levels $\left[\mathrm{m}=10.0_{(0.4)}\right.$ per sampling block $]$. The term "plume" describes the basic plume-like shape of these zones where Fos expression was increased around the microinjection site (compared with controls). Thus, DAMGO microinjections in the central nucleus of amygdala produced roughly spherical Fos plumes, with a total volume of tissue of $\sim 0.43 \mathrm{~mm}^{3}(0.47 \mathrm{~mm}=$ mean radius, used for symbols in maps).

Conversely, muscimol microinjections suppressed Fos expression to below half the spontaneous control levels of uninjected brains in a large zone surrounding the microinjection center $\left[1.9_{(0.16)}\right.$ Fos-positive cells per $0.125 \mathrm{~mm}^{2}$ sampling block in BMA and MeA]. Such inhibitory zones of local Fos suppression have previously been called "antiplumes" (Smith and Berridge, 2007). Typically, a large antiplume of inhibition surrounded a small excitatory center near the microinjection center that constituted $<20 \%$ of its total volume $\left[7.2_{(0.4)}\right.$ cells per sampling block]. Overall, the total volume of muscimol-induced neuronal modulation (central Fos plume + outer Fos antiplume) was 0.21 $\mathrm{mm}^{3}$, which was used for mapping symbols $(0.37 \mathrm{~mm}$ total radius). Examples of Fos plumes are shown in Figure 2 and supplemental Figure 5 (available at www.jneurosci.org as supplemental material), and complete Fos plume radii and estimated volumes are listed in supplemental Table 1 (available at www.jneurosci. org as supplemental material).

The entire volume of the unilateral CeA nucleus was estimated to be $\sim 1.27 \mathrm{~mm}^{3}(\sim 2 \mathrm{~mm}$ AP $\times$ between 0.4 and $1.3 \mathrm{~mm} \mathrm{DV}$ and $\mathrm{ML}$ ), and we calculated the average DAMGO plume center of intense Fos elevation to fill $\sim 2.4 \%$ of CeA volume, whereas its outer plume of moderate Fos filled $\sim 43 \%$ of CeA volume. The largest muscimol outer plume (actually an inhibitory antiplume) similarly filled $\sim 36 \%$ of CeA volume. Beyond the CeA, the larger amygdala complex (including basolateral, medial, and basal nuclei as well as central nucleus) was estimated to be $\sim 8.7 \mathrm{~mm}^{3}$, which implied that each outer DAMGO or muscimol plume/ antiplume filled $\sim 5-6 \%$ of the entire amygdala complex (amygdala between AP levels 1.32 and 3.36 caudal of bregma; size $=\sim 2$ $\mathrm{mm}$ AP $\times 1.4-2.9 \mathrm{~mm} \mathrm{DV}$ and $\mathrm{ML})$.

On the basis of these measurements and on site placements confirmed histologically in rats used for behavioral experiments, we calculated that $94 \%$ of rats in behavioral experiments had Fos plumes mostly contained within the CeA $(n=79)$. DAMGO injections had the strongest effects on prepotent CS consummatory behaviors when microinjections were centered directly into the CeA, as opposed to just outside it. For example, placements that would produce plumes contained completely within the CeA enhanced nibbles and sniffs to $>160 \%$ of vehicle levels [vehicle $\mathrm{m}_{(\mathrm{SEM})}=3.4_{(0.5)}$, DAMGO $\left.\mathrm{m}=5.8_{(0.6)} ; t_{(8)}=5.1, p=0.001\right]$. Intense DAMGO Fos plumes were likely entirely contained within the CeA on at least one side for $69 \%$ of rats $(n=58)$ and on both sides for $57 \%$ of rats $(n=48)$. On average, $74 \%$ of each CeA-centered DAMGO plume would have stayed entirely within the boundaries of CeA, including nearly all of the most intense Fos activation zones. The remainder of peripheral plumes likely penetrated the basolateral (BLA; 33\%), basomedial (BMA; 25\%), or medial (MeA; 25\%) nuclei of amygdala, which are also implicated in motivation or learning. In our site control results, placements for which the plumes were calculated to be mostly outside the CeA failed to enhance consummatory behaviors $[n=15$; $108 \%$ of vehicle; vehicle $\mathrm{m}=4.3_{(0.5)}$; DAMGO $\mathrm{m}=4.4_{(0.5)} ; t_{(14)}$ $=0.1$, n.s. $]$. We note that this does not exclude participatory roles in normal reward-related motivation and learning for BLA or BMA, which have been suggested by lesion studies (Holland et al., 2002; Everitt et al., 2003; See et al., 2003; McDannald et al., 2004; Corbit and Balleine, 2005; Petrovich and Gallagher, 2007) but suggest only that opioid stimulation of CeA is sufficient to enhance incentive salience. Future studies would be needed for a more complete comparison map of amygdala nuclei.

In addition, we again found some evidence for localization of function within subregions of CeA itself. Only DAMGO sites in the anterior half of the CeA produced strong enhancements of consummatory sniffs and nibbles of the sucrose-delivering $\mathrm{CS}_{\text {cup }}$ in $\mathrm{CS}_{\text {cup }}$-preferring rats (between -1.4 and $-2.04 \mathrm{~mm}$ caudal of bregma; $132 \%$ of vehicle day consummatory behavior). Furthermore, DAMGO at sites in the posterior half of the CeA actually decreased consummatory behaviors directed toward the $\mathrm{CS}_{\text {cup }}$ ( sites between -2.04 and $-3.4 \mathrm{~mm}$ caudal of bregma: $87 \%$ of vehicle day, $t_{(4)}=2.9, p<0.05$; anterior vs posterior difference, $F_{(1,7)}$ $=7.7, p<0.05]$. In contrast, the entire CeA appeared to support enhancements of CS+ lever interactions in rats that preferred this CS, suggesting a possible interaction between cue prepotency and subregional differences that deserves further exploration.

\section{Discussion}

Here, we report that opioid stimulation of the CeA intensified and focused incentive salience to make one reward cue more "wanted" than others. CeA microinjections of the $\mu$ opioid agonist DAMGO increased appetitive and consummatory behaviors toward each individual's prepotent cue (selected based on pavlovian learning and individual factors), without amplifying the attractiveness of other cues. Normally, nibbling and sniffing behaviors are directed at food itself, but here, they were directed at a starkly artificial metal CS that became a prepotent incentive cue (Jenkins and Moore, 1973; Davey and Cleland, 1982) and were intensified to frenzied-appearing levels by CeA DAMGO (Fig. 3; Video 1, available at www.jneurosci.org as supplemental material). Conversely, CeA inactivation by muscimol microinjection dramatically reduced these appetitive and consummatory behaviors. Thus, we conclude that CeA bidirectionally modulates the amplitude of incentive salience, making particular reward cues more or less "wanted." Focused CS "wanting" may often guide adaptive behavior toward a reward, but at intense levels may also have maladaptive consequences, as in addiction.

Here, each rat had its own individual prepotent CS - the target it preferentially approached and orally manipulated with intense nibbling and sniffing behaviors. For some, the prepotent cue-triggered incentive target was the sucrose-predictive CS+ lever, whereas for others it was the sucrose-delivering $\mathrm{CS}_{\text {cup }}$. In both cases, CeA opioid activation made only the already prepotent CS more powerfully able to pull in appetitive and consummatory behavior as a "motivational magnet." The other CSs in the chamber were typically not enhanced. Even for the few rats 
that ordinarily split their nibbles and sniffs between both CSs, CeA DAMGO enhanced the incentive salience of only one. Thus, CeA opioid activation appears to magnify and focus incentive salience predominantly on one cue at a time.

We do not suggest that rats confused their prepotent cue with the associated food reward (stimulus substitution), since their consummatory behaviors toward their CS+ lever or $\mathrm{CS}_{\text {cup }}$ dish cue remained sensitive to their physical metal features. For example, DAMGO stimulated only the nibble and sniff behaviors that constitute the initial phase of CS consummatory bouts, but never the terminal phase of slower bites that lead to actual swallowing of food UCSs. Furthermore, the consummatory behaviors toward the CS were distorted in that DAMGO in CeA actually drove CS nibbles and sniffs to higher levels than were usually displayed toward actual food pellets (while not enhancing consumption bites). This suggests that physical CS features continued to influence the expression of motivated behavior-just as UCS features can determine whether behavior directed at pavlovian cues is ingestive, social, sexual, or another motivation type (Jenkins and Moore, 1973; Lajoie and Bindra, 1976; Timberlake and Lucas, 1985; Tomie, 1996; Uslaner et al., 2006).

We note that our incentive salience interpretation of CeA opioid enhancement goes beyond interpretations of cue-directed behaviors as merely approach or attentional sign-tracking of pavlovian stimuli (Kaye and Pearce, 1984; Cardinal et al., 2002; Everitt et al., 2003; McDannald et al., 2004; Day et al., 2006; ElAmamy and Holland, 2007), although these interpretations are not mutually exclusive. Analogously, human drug addicts do notice and approach drug stimuli, but also find those stimuli motivationally compelling, making the addiction difficult to give up. Similarly, the rats' intense, frenzy-like bursts of consummatory behaviors toward their prepotent CS seems best explained with a motivation-based incentive salience framework. That framework suggests that CeA DAMGO made the prepotent cue take on stronger UCS-appropriate incentive properties, and so become more wanted in a food-like manner (although not fully substituting for food).

\section{CeA opioids enhance phasic temporal peaks of "wanting"}

DAMGO was constantly present in CeA and only gradually declined during test sessions, but responses toward the reward cue occurred as transient $8 \mathrm{~s}$ bursts that were closely time-locked to insertions of the CS + lever. This phasic pattern of enhancement was not simply the result of CS availability, since it applied even to the $\mathrm{CS}_{\text {cup }}$, which was constantly present. Rather, the phasic pattern reflects a synergistic or multiplicative interaction in generating incentive salience between (1) mesocorticolimbic activation (relatively constant) and (2) presence of an associative CS for reward (phasic). That is, according to the incentive salience hypothesis, limbic activation multiplies the incentive value of a perceived CS, producing "wanting" in pulses that come and go with the CS rather than as a steady, constant drive (Wyvell and Berridge, 2001; Tindell et al., 2005). A related measure of cuetriggered incentive salience is pavlovian to instrumental transfer (PIT), which is attenuated by CeA lesions (Hall et al., 2001; Holland and Gallagher, 2003; Corbit and Balleine, 2005). If CeA opioids indeed enhance incentive salience "wanting," then it can be predicted that CeA DAMGO microinjection should also amplify cue-triggered "wanting" for a UCS reward in a phasic-pulsed manner in PIT, similar to its amplification of CS incentive motivation here.

\section{Anatomical localization of function}

Beyond the amygdala, incentive salience is mediated by larger mesocorticolimbic reward circuits, including nucleus accumbens dopamine, ventral pallidum, and other structures (Wyvell and Berridge, 2000; Zhang et al., 2003; Tindell et al., 2005; Day et al., 2007; Volkow et al., 2007; Di Ciano, 2008). CeA opioid activation likely recruited these other components (Phillips et al., 2008). Additionally, DAMGO microinjections here sometimes spread to BLA or medial amygdala (MeA), which have roles in reward learning and motivation (Cardinal et al., 2002; Everitt et al., 2003; See et al., 2003; McDannald et al., 2004; Corbit and Balleine, 2005), and are even more important than CeA for some cueevoked consummatory behaviors, such as eating (Holland et al., 2002; Petrovich and Gallagher, 2007). Future studies will be needed to clarify the relative roles of CeA versus other amygdala nuclei in cue-triggered incentive salience, and results may depend on the conditioned behavioral response (e.g., CS autoshaping or cue-triggered UCS intake), type of neural manipulation (pharmacological stimulation or lesion), and functional consequence (stimulation of motivation or attenuation) examined in each study.

Within the CeA, some evidence indicated that a "hot spot" may exist within an anterior subregion for amplifying CSdirected motivation. DAMGO microinjections in the rostral onethird $\left(\sim 0.4 \mathrm{~mm}^{3}\right)$ of the CeA nearly doubled consummatory behaviors, whereas more caudal injections actually caused reductions below control levels. We note that the rostral CeA is reported to differ in several neurobiological ways from caudal CeA, which might be related to localization of function for motivational effects (Zardetto-Smith and Gray, 1990; Bernard et al., 1993; Shammah-Lagnado et al., 2001; Jolkkonen et al., 2002).

\section{Fear versus feeding effects in CeA}

CeA manipulations sometimes appeared to elicit fearful or defensively motivated behavior in addition to appetitive motivation. For example, muscimol inactivation of $\mathrm{CeA}$ caused defensive treading in most rats when they were tested in chambers containing corncob bedding suitable for throwing and pushing. Defensive treading is associated with fearful motivation (Owings and Coss, 1977; Treit et al., 1981; Reynolds and Berridge, 2008) and consists of forceful pushing of bedding toward a threat (here, typically toward the experimenter visible beyond the transparent wall of the chamber). CeA opioid activation also elicited defensive behavior from some rats, which was sometimes even directed toward the same food pellet the rat ate a few seconds before or later. Fearful behavior appeared independently of appetitive motivation (i.e., treading accompanied increased food intake after DAMGO, and decreased food intake after muscimol). These mixtures of fear and feeding motivation might reflect ambivalence in motivational salience, consistent with the possibility that the generation of desire and dread may share some underlying mesocorticolimbic mechanisms (Salamone, 1994; Levita et al., 2002; Kapur, 2003; Faure et al., 2008; Reynolds and Berridge, 2008).

\section{Addiction and targeted incentive salience}

Drug addiction and other compulsive motivational disorders often involve excessive motivational attractiveness of pavlovian CSs. For example, some cocaine addicts "chase ghosts," drawn by their attraction to small white pebbles (Rosse et al., 1993), which could result from sensitized levels of "wanting" targeted on stimuli that resemble cocaine CSs (Robinson and Berridge, 1993). The incentive-sensitization theory of addiction posits that excessive motivational salience of drug cues contributes to cueinduced craving and relapse, even after long abstinence from drugs (Robinson and Berridge, 1993, 2008). Our results may open a way to explain a puzzle in cued relapse: how incentive- 
sensitization can specifically amplify "wanting" for drug CSs without amplifying "wanting" for other CSs or rewards (Vanderschuren and Everitt, 2005). At least in principle, our findings indicate that particular reward cues can be selectively targeted with enhanced incentive salience in a "winner take all" manner. In this case, enhancement resulted from activation of CeA opioid neurotransmission, and we note that drug cues elicit amygdala activation (including opioid activation) in human addicts (Grant et al., 1996; Franklin et al., 2007; Scott et al., 2007). Furthermore, our results also suggest drug priming could further enhance the incentive salience of prepotent cues, as DAMGO microinjections did here, thereby intensifying the urge to binge in an addict who had tried to take "just a little" drug. Similar CeA mechanisms, if endogenously activated to aberrantly focus incentive salience on cues for food, money, or other rewards, might also participate in binge eating, gambling, and other compulsive disorders.

\section{References}

Baldo BA, Alsene KM, Negron A, Kelley AE (2005) Hyperphagia induced by $\mathrm{GABA}_{\mathrm{A}}$ receptor-mediated inhibition of the nucleus accumbens shell: dependence on intact neural output from the central amygdaloid region. Behav Neurosci 119:1195-1206.

Balleine BW, Killcross S (2006) Parallel incentive processing: an integrated view of amygdala function. Trends Neurosci 29:272-279.

Beaver JD, Lawrence AD, van Ditzhuijzen J, Davis MH, Woods A, Calder AJ (2006) Individual differences in reward drive predict neural responses to images of food. J Neurosci 26:5160-5166.

Bernard JF, Alden M, Besson JM (1993) The organization of the efferent projections from the pontine parabrachial area to the amygdaloid complex: a Phaseolus vulgaris leucoagglutinin (PHA-L) study in the rat. J Comp Neurol 329:201-229.

Berridge K (2001) Reward learning: reinforcement, incentives, and expectations. In: Psychology of learning and motivation (Medin DL, ed), pp 223-278. New York: Academic.

Berridge KC (2007) The debate over dopamine's role in reward: the case for incentive salience. Psychopharmacology 191:391-431.

Bindra D (1978) How adaptive behavior is produced: a perceptualmotivation alternative to response reinforcement. Behav Brain Sci 1:41-91.

Boakes R (1977) Performance on learning to associate a stimulus with positive reinforcement. In: Operant-pavlovian interactions (Davis H, Hurwitz H, eds), pp 67-97. Hillsdale, NJ: Lawrence Erlbaum Associates.

Burns M, Domjan M (1996) Sign tracking versus goal tracking in the sexual conditioning of male Japanese quail (Coturnix japonica). J Exp Psychol Anim Behav Process 22:297-306.

Cardinal RN, Parkinson JA, Lachenal G, Halkerston KM, Rudarakanchana N, Hall J, Morrison CH, Howes SR, Robbins TW, Everitt BJ (2002) Effects of selective excitotoxic lesions of the nucleus accumbens core, anterior cingulate cortex, and central nucleus of the amygdala on autoshaping performance in rats. Behav Neurosci 116:553-567.

Corbit LH, Balleine BW (2005) Double dissociation of basolateral and central amygdala lesions on the general and outcome-specific forms of pavlovian-instrumental transfer. J Neurosci 25:962-970.

Davey GC, Cleland GG (1982) Topography of signal-centered behavior in the rat: effects of deprivation state and reinforcer type. J Exp Anal Behav 38:291-304.

Day JJ, Wheeler RA, Roitman MF, Carelli RM (2006) Nucleus accumbens neurons encode pavlovian approach behaviors: evidence from an autoshaping paradigm. Eur J Neurosci 23:1341-1351.

Day JJ, Roitman MF, Wightman RM, Carelli RM (2007) Associative learning mediates dynamic shifts in dopamine signaling in the nucleus accumbens. Nat Neurosci 10:1020-1028.

Di Ciano P (2008) Facilitated acquisition but not persistence of responding for a cocaine-paired conditioned reinforcer following sensitization with cocaine. Neuropsychopharmacology 33:1426-1431.

Di Ciano P, Everitt BJ (2004) Direct interactions between the basolateral amygdala and nucleus accumbens core underlie cocaine-seeking behavior by rats. J Neurosci 24:7167-7173.

Di Ciano P, Everitt BJ (2005) Neuropsychopharmacology of drug seeking: insights from studies with second-order schedules of drug reinforcement. Eur J Pharmacol 526:186-198.

El-Amamy H, Holland PC (2007) Dissociable effects of disconnecting amygdala central nucleus from the ventral tegmental area or substantia nigra on learned orienting and incentive motivation. Eur J Neurosci 25:1557-1567.

Everitt BJ, Cardinal RN, Parkinson JA, Robbins TW (2003) Appetitive behavior: impact of amygdala-dependent mechanisms of emotional learning. Ann N Y Acad Sci 985:233-250.

Faure A, Reynolds SM, Richard JM, Berridge KC (2008) Mesolimbic dopamine in desire and dread: enabling motivation to be generated by localized glutamate disruptions in nucleus accumbens. J Neurosci 28:7184-7192.

Flagel SB, Watson SJ, Robinson TE, Akil H (2007) Individual differences in the propensity to approach signals vs goals promote different adaptations in the dopamine system of rats. Psychopharmacology 191:599-607.

Flagel SB, Watson SJ, Akil H, Robinson TE (2008) Individual differences in the attribution of incentive salience to a reward-related cue: influence on cocaine sensitization. Behav Brain Res 186:48-56.

Flagel SB, Akil H, Robinson TE (2009) Individual differences in the attribution of incentive salience to reward-related cues: implications for addiction. Neuropharmacology 56 [Suppl 1]:139-148.

Franklin TR, Wang Z, Wang J, Sciortino N, Harper D, Li Y, Ehrman R, Kampman K, O’Brien CP, Detre JA, Childress AR (2007) Limbic activation to cigarette smoking cues independent of nicotine withdrawal: a perfusion fMRI study. Neuropsychopharmacology 32:2301-2309.

Gallagher M, Schoenbaum G (1999) Functions of the amygdala and related forebrain areas in attention and cognition. Ann NY Acad Sci 877:397-411.

Giraudo SQ, Billington CJ, Levine AS (1998) Effects of the opioid antagonist naltrexone on feeding induced by DAMGO in the central nucleus of the amygdala and in the paraventricular nucleus in the rat. Brain Res 782:18-23.

Glass MJ, Billington CJ, Levine AS (2000) Naltrexone administered to central nucleus of amygdala or PVN: neural dissociation of diet and energy. Am J Physiol Regul Integr Comp Physiol 279:R86-R92.

Gosnell BA (1988) Involvement of $\mu$ opioid receptors in the amygdala in the control of feeding. Neuropharmacology 27:319-326.

Grant S, London ED, Newlin DB, Villemagne VL, Liu X, Contoreggi C, Phillips RL, Kimes AS, Margolin A (1996) Activation of memory circuits during cue-elicited cocaine craving. Proc Natl Acad Sci USA 93:12040-12045.

Hall J, Parkinson JA, Connor TM, Dickinson A, Everitt BJ (2001) Involvement of the central nucleus of the amygdala and nucleus accumbens core in mediating pavlovian influences on instrumental behaviour. Eur J Neurosci 13:1984-1992.

Hearst E, Jenkins HM (1974) Sign tracking: the stimulus-reinforcer relation and directed action. In: Monograph of the Psychonomic Society. Austin, TX: The Psychonomic Society.

Holland PC (1977) Conditioned stimulus as a determinant of the form of the pavlovian conditioned response. J Exp Psychol Anim Behav Process 3:77-104.

Holland PC, Gallagher M (2003) Double dissociation of the effects of lesions of basolateral and central amygdala on conditioned stimulus-potentiated feeding and pavlovian-instrumental transfer. Eur J Neurosci 17:1680-1694.

Holland PC, Gallagher M (2004) Amygdala-frontal interactions and reward expectancy. Curr Opin Neurobiol 14:148-155.

Holland PC, Petrovich GD, Gallagher M (2002) The effects of amygdala lesions on conditioned stimulus-potentiated eating in rats. Physiol Behav 76:117-129.

Jenkins HM, Moore BR (1973) The form of the auto-shaped response with food or water reinforcers. J Exp Anal Behav 20:163-181.

Jolkkonen E, Miettinen R, Pikkarainen M, Pitkänen A (2002) Projections from the amygdaloid complex to the magnocellular cholinergic basal forebrain in rat. Neuroscience 111:133-149.

Kapur S (2003) Psychosis as a state of aberrant salience: a framework linking biology, phenomenology, and pharmacology in schizophrenia. Am J Psychiatry 160:13-23.

Kaye H, Pearce JM (1984) The strength of the orienting response during pavlovian conditioning. J Exp Psychol Anim Behav Process 10:90-109.

Kim EM, Quinn JG, Levine AS, O’Hare E (2004) A bidirectional $\mu$-opioid- 
opioid connection between the nucleus of the accumbens shell and the central nucleus of the amygdala in the rat. Brain Res 1029:135-139.

Lajoie J, Bindra D (1976) An interpretation of autoshaping and related phenomena in terms of stimulus-incentive contingencies alone. Rev Can Psychol 30:157-173.

Levita L, Dalley JW, Robbins TW (2002) Nucleus accumbens dopamine and learned fear revisited: a review and some new findings. Behav Brain Res 137:115-127.

Mahler SV, Smith KS, Berridge KC (2007) Endocannabinoid hedonic hotspot for sensory pleasure: anandamide in nucleus accumbens shell enhances "liking" of a sweet reward. Neuropsychopharmacology 32:2267-2278.

Maren S, Yap SA, Goosens KA (2001) The amygdala is essential for the development of neuronal plasticity in the medial geniculate nucleus during auditory fear conditioning in rats. J Neurosci 21:RC135.

McDannald M, Kerfoot E, Gallagher M, Holland PC (2004) Amygdala central nucleus function is necessary for learning but not expression of conditioned visual orienting. Eur J Neurosci 20:240-248.

Morris JS, Dolan RJ (2001) Involvement of human amygdala and orbitofrontal cortex in hunger-enhanced memory for food stimuli. J Neurosci 21:5304-5310.

Owings D, Coss R (1977) Snake mobbing by California ground squirrels: adapative variation and ontogeny. Behaviour 62:50-69.

Paré D, Quirk GJ, Ledoux JE (2004) New vistas on amygdala networks in conditioned fear. J Neurophysiol 92:1-9.

Paxinos G, Watson C (2007) The rat brain in stereotaxic coordinates, Ed 6 . Amsterdam: Elsevier.

Peciña S, Berridge KC (2000) Opioid site in nucleus accumbens shell mediates eating and hedonic "liking" for food: map based on microinjection Fos plumes. Brain Res 863:71-86.

Peciña S, Berridge KC (2005) Hedonic hot spot in nucleus accumbens shell: where do $\mu$-opioids cause increased hedonic impact of sweetness? J Neurosci 25:11777-11786.

Peterson GB, Ackilt JE, Frommer GP, Hearst ES (1972) Conditioned approach and contact behavior toward signals for food or brain-stimulation reinforcement. Science 177:1009-1011.

Petrovich GD, Gallagher M (2007) Control of food consumption by learned cues: a forebrain-hypothalamic network. Physiol Behav 91:397-403.

Phelps EA, LeDoux JE (2005) Contributions of the amygdala to emotion processing: from animal models to human behavior. Neuron 48:175-187.

Phillips AG, Vacca G, Ahn S (2008) A top-down perspective on dopamine, motivation and memory. Pharmacol Biochem Behav 90:236-249.

Reynolds SM, Berridge KC (2001) Fear and feeding in the nucleus accumbens shell: rostrocaudal segregation of GABA-elicited defensive behavior versus eating behavior. J Neurosci 21:3261-3270.

Reynolds SM, Berridge KC (2008) Emotional environments retune the valence of appetitive versus fearful functions in nucleus accumbens. Nat Neurosci 11:423-425.

Robbins TW, Everitt BJ (1996) Neurobehavioural mechanisms of reward and motivation. Curr Opin Neurobiol 6:228-236.

Robinson TE, Berridge KC (1993) The neural basis of drug craving: an incentive-sensitization theory of addiction. Brain Res Brain Res Rev $18: 247-291$

Robinson TE, Berridge KC (2008) Review: the incentive sensitization theory of addiction: some current issues. Philos Trans R Soc Lond B Biol Sci 363:3137-3146

Rodgers RJ, Cao BJ, Dalvi A, Holmes A (1997) Animal models of anxiety: an ethological perspective. Braz J Med Biol Res 30:289-304.

Rosse RB, Fay-McCarthy M, Collins JP Jr, Risher-Flowers D, Alim TN, Deutsch SI (1993) Transient compulsive foraging behavior associated with crack cocaine use. Am J Psychiatry 150:155-156.

Salamone JD (1994) The involvement of nucleus accumbens dopamine in appetitive and aversive motivation. Behav Brain Res 61:117-133.
Scott DJ, Domino EF, Heitzeg MM, Koeppe RA, Ni L, Guthrie S, Zubieta JK (2007) Smoking modulation of $\mu$-opioid and dopamine D2 receptormediated neurotransmission in humans. Neuropsychopharmacology 32:450-457.

See RE, Fuchs RA, Ledford CC, McLaughlin J (2003) Drug addiction, relapse, and the amygdala. Ann N Y Acad Sci 985:294-307.

Shammah-Lagnado SJ, Alheid GF, Heimer L (2001) Striatal and central extended amygdala parts of the interstitial nucleus of the posterior limb of the anterior commissure: evidence from tract-tracing techniques in the rat. J Comp Neurol 439:104-126.

Smith KS, Berridge KC (2005) The ventral pallidum and hedonic reward: neurochemical maps of sucrose "liking" and food intake. J Neurosci 25:8637-8649.

Smith KS, Berridge KC (2007) Opioid limbic circuit for reward: interaction between hedonic hotspots of nucleus accumbens and ventral pallidum. J Neurosci 27:1594-1605.

Timberlake W, Grant DL (1975) Autoshaping in rats to the presentation of another rat predicting food. Science 190:690-692.

Timberlake W, Lucas GA (1985) The basis of superstitious behavior: chance contingency, stimulus substitution, or appetitive behavior? J Exp Anal Behav 44:279-299.

Tindell AJ, Berridge KC, Zhang J, Peciña S, Aldridge JW (2005) Ventral pallidal neurons code incentive motivation: amplification by mesolimbic sensitization and amphetamine. Eur J Neurosci 22:2617-2634.

Toates F (1986) Motivational systems. Cambridge, UK: Cambridge UP.

Tomie A (1996) Locating reward cue at response manipulandum (CAM) induces symptoms of drug abuse. Neurosci Biobehav Rev 20:505-535.

Tomie A, Grimes KL, Pohorecky LA (2008) Behavioral characteristics and neurobiological substrates shared by pavlovian sign-tracking and drug abuse. Brain Res Rev 58:121-135

Treit D, Pinel JP, Fibiger HC (1981) Conditioned defensive burying: a new paradigm for the study of anxiolytic agents. Pharmacol Biochem Behav 15:619-626.

Uslaner JM, Acerbo MJ, Jones SA, Robinson TE (2006) The attribution of incentive salience to a stimulus that signals an intravenous injection of cocaine. Behav Brain Res 169:320-324.

Valenstein ES, Cox VC, Kakolewski JW (1970) Reexamination of the role of the hypothalamus in motivation. Psychol Rev 77:16-31.

Vanderschuren LJ, Everitt BJ (2005) Behavioral and neural mechanisms of compulsive drug seeking. Eur J Pharmacol 526:77-88.

Volkow ND, Fowler JS, Wang GJ, Swanson JM, Telang F (2007) Dopamine in drug abuse and addiction: results of imaging studies and treatment implications. Arch Neurol 64:1575-1579.

Wilensky AE, Schafe GE, LeDoux JE (2000) The amygdala modulates memory consolidation of fear-motivated inhibitory avoidance learning but not classical fear conditioning. J Neurosci 20:7059-7066.

Wyvell CL, Berridge KC (2000) Intra-accumbens amphetamine increases the conditioned incentive salience of sucrose reward: enhancement of reward "wanting" without enhanced "liking" or response reinforcement. J Neurosci 20:8122-8130.

Wyvell CL, Berridge KC (2001) Incentive sensitization by previous amphetamine exposure: increased cue-triggered "wanting" for sucrose reward. J Neurosci 21:7831-7840.

Zardetto-Smith AM, Gray TS (1990) Organization of peptidergic and catecholaminergic efferents from the nucleus of the solitary tract to the rat amygdala. Brain Res Bull 25:875-887.

Zhang M, Balmadrid C, Kelley AE (2003) Nucleus accumbens opioid, GABaergic, and dopaminergic modulation of palatable food motivation: contrasting effects revealed by a progressive ratio study in the rat. Behav Neurosci 117:202-211.

Zimmerman JM, Rabinak CA, McLachlan IG, Maren S (2007) The central nucleus of the amygdala is essential for acquiring and expressing conditional fear after overtraining. Learn Mem 14:634-644. 